\title{
DYNAMIC PROGRAMMING FOR CONTROLLED MARKOV FAMILIES: ABSTRACTLY AND OVER MARTINGALE MEASURES*
}

\author{
GORDAN ŽITKOVIĆ ${ }^{\dagger}$
}

\begin{abstract}
We describe an abstract control-theoretic framework in which the validity of the dynamic programming principle can be established in continuous time by a verification of a small number of structural properties. As an application we treat several cases of interest, most notably the lower-hedging and utility-maximization problems of financial mathematics both of which are naturally posed over "sets of martingale measures".
\end{abstract}

Key words. dynamic programming, financial mathematics, lower hedging, Markov processes, optimal stochastic control, utility maximization

AMS subject classifications. 93E20, 60G44, 60J25, 91G80,

1. Introduction. The goal of this article is to prove the dynamic programming principle (DPP in the sequel) for a class of stochastic control problems in a continuous-time Markov-like environment, including two fundamental problems in financial mathematics defined over or parametrized by a set of "martingale" measures. To this end, we introduce a flexible abstract framework in which, we hope, other problems can be treated as well.

The history of the DPP is fascinating; it starts with the work of Wald [44] and Bellman [5], although the idea of the reduction of a complicated sequential problem to a family of simpler ones is undoubtedly much older. A rigorous discrete-time theory focusing on intricate measurability issues started with the work of Blackwell and others (see, e.g., $[10,11,40,12,13,7]$; we refer the reader to [8] for further bibliographical information and a detailed discussion). It was already known to these authors that - unlike in the setting of deterministic optimal control, where the validity of the DPP is easier to establish - the stochastic version of the DPP comes with a number of additional subtleties and requires a much more delicate treatment. For this reason, two main schools of thought dominate the control-theoretic literature. In one of them, great importance is given to a rigorous derivation of an appropriate version of the DPP. The other, however, treats the very need for a proof of the DPP as a mathematical pedantry and sees it as an evidently correct general principle.

In order to provide a suitable general theoretical foundation in continuous time, and, in the endgame, a practical convergence of the two approaches, a flurry of activity over the last decades expanded tremendously our understanding of continuous-time DPP in a variety of settings (see, e.g., [22], [14], [26], [37], [38], [18], [17], [15], etc.).

${ }^{*}$ The author would like to thank Gerard Brunick, Kasper Larsen and Mihai Sîrbu for numerous and helpful discussions on the subject of dynamic programming. Careful reading by the anonymous referees and the associate editor of the original manuscript, as well as their numerous suggestions for improvement, is gratefully acknowledged. This material is based upon work supported by the National Science Foundation under Grants No. DMS-0706947 (2010 - 2015) and Grant No. DMS1107465 (2012 - 2017). Any opinions, findings and conclusions or recommendations expressed in this material are those of the author(s) and do not necessarily reflect the views of the National Science Foundation (NSF)

${ }^{\dagger}$ Department of Mathematics, The University of Texas at Austin 
Independently of the present paper, and with a different point of view, the authors of [23] and [24] also provide an abstract approach to the dynamic programming and give a thorough treatment of descriptive set-theoretic machinery which underlies it. Moreover, a similar descriptive-set-theoretic idea have recently been used successfully in the context of model uncertainty and nonlinear expectations (see [32], [33] and [16]), with the setting and technique, especially in [33], similar to that of parts of Sections 2.1 and 3.2 of the present paper.

A different point of view, called the "Stochastic Perron Method", initiated by Bayraktar and Sirbu (see [3], [2] and [4]) circumvents the use of the formal DPP altogether and derives verification results for the corresponding HJB equations directly under natural condition on the equation itself.

1.1. Weakly-constrained problems and the convenience of the weak formulation. While quite general and extremely useful in their own domains, none of the existing results seem to apply to the general Markovian versions of the problems of lower hedging or utility maximization, often seen as fundamental in the field of financial mathematics. These problems are naturally defined over sets of equivalent local-martingale measures and, depending on the particular setting, do not admit a naive translation into the classical control milieu. Indeed, they usually come in the "weakly-constrained" form: when framed in the classical stochastic-control language, the set of admissible controls is unconstrained locally, but still required to consist of processes which, when acted upon by a stochastic-exponential type operator, yield uniformly-integrable (as opposed to merely local) martingales. This difficulty vanishes when one chooses to view these problems in their (we dare say, even more natural) weak formulation, and works with sets of probability measures rather than control processes right from the start. The weak approach is certainly not new in stochastic control theory; the first uses of a "controlled martingale problem" go back at least to [9]. Many other authors (see [45] and [27] and the references therein) use similar concepts under different names, such as the "weak formulation".

An added technical benefit of the weak approach is the complete avoidance of subtle measure-theoretic difficulties (mostly dealing with the stochastic integration) inherent to the DPP-based treatment of strongly formulated problems. In fact, it could be argued that the entire "philosophy" of our approach is based on this fact: the descriptive-set-theoretic and topological framework of classical discrete-time optimal control and the analytical and process-theoretic framework of the contemporary stochastics do not seem to play well together. For example, the filtration completion makes a tractable stochastic-integration theory possible, while simultaneously destroying the countable-generation property.

The strong formulation - where the controls and the noise inhabit a fixed filtered probability space - may seem to be more amenable to concatenation (the basic construction in any DPP treatment). The message we are sending here is that, from the analytical point of view, measures are easier to work with, and just as easy to concatenate. This perspective aligns well with the mental model often present in the classical gambling theory (see [31]), wherein the player (controller) chooses a "gambling house", i.e., one among possibly many available probability measures to govern the future evolution of the state process. Clearly, the difference lies mostly in inter- 
pretation, but, as we hope our results demonstrate, it can be an important one.

1.2. An abstract version of the DPP. To implement the conceptual framework described above, we introduce a structure, called the controlled Markov family, reminiscent of, and modeled on, a classical Markov family associated to a Markov process. It consists of a family of sets of probability measures, one for each point of the state space $E$, with the interpretation that the probability measures available at $x \in E$ can be used by the controller in order to steer the system so as to minimize the expected value of the given cost functional. These probability measures are defined on the set $D_{E}$ of RCLL trajectories and do not necessarily form a Markov family, or correspond to a Markov process on $E$. In fact, our main theorem can be understood as a description of how close to "being Markov" our families of sets of probability measures (one for each $x \in E$ ) must be in order for the DPP to hold. Put yet differently, we are interested in a useful generalization of Chapman-Kolmogorov equations to the set-valued case.

It turns out that two natural requirements, namely concatenability (closure under concatenation) and analyticity, lead to a convenient definition. The first makes sure that the controller can change his/her mind midstream and switch to any measure available at the current state. The second condition is of purely technical nature, and imposes a minimal degree of regularity on how the family of available probability laws changes from point to point. To understand it better, let us mention that analyticity directly implies that the value function is upper semi-analytic, which, in turn, makes it universally measurable. As observed in [36], universal measurability (i.e., measurability with respect to a completion of the Borel $\sigma$-algebra under any Borel probability measure) is about the weakest property one can require from the value function for even the formulation of the DPP to make sense. Indeed, the righthand side of a typical DPP statement will involve the expectation (an integral) of the value function applied to a random variable, which, without a minimal degree of measurability, cannot even be defined without running into serious conceptual difficulties.

While the value functions associated with controlled Markov processes already possess many good properties and satisfy "half" of the DPP, another condition in a sense dual to concatenability - is needed for the full DPP to hold. Named (approximate) disintegrability, it postulates that, if required to switch to an admissible control, the controller can oblige at any point in time and still remain (nearly) optimal. Our main abstract result states that a controlled Markov family with the additional property of disintegrability defines a value function for which the full DPP holds and extends the classical descriptive-set-theoretic approach of discretetime stochastic optimal control to continuous time with Markovian-like dynamics. As we explain it in more detail in subsection 2.4, part (1), that canonical Feller families (and virtually all other Markov families under minimal regularity conditions), when understood as controlled Markov families with singleton control sets, automatically satisfy the three key properties of analyticity, concatenability and disintegrability.

It is important to make it clear that our main abstract result - Theorem 2.4 together with the techniques used in its proof, is quite similar to some of the related results found throughout the literature (some of them going back to Blackwell). On 
the other hand, its scope and the level of abstraction are new and seem to be unavailable; its value lies primarily in its applicability in specific situations, as explained below.

1.3. Examples and applications to financial mathematics. After the main abstract theorem and a practical sufficient condition for its assumptions to hold, the paper treats several examples in various degrees of detail. We start by showing that many (uncontrolled) Markov families - such as the canonical RCLL Markov families - naturally satisfy the conditions of concatenability, disintegrability and analyticity, in a rather trivial way. We comment, though, that the families obtained by solving martingale problems with multiple solutions furnish a less trivial example: the family of sets of all weak solutions of a martingale problem will form a controlled Markov family under the right regularity conditions, too. Next, we turn to a bird's-eye discussion of how one can interpret control problems in the classical strong formulation as controlled Markov families, and what structure is needed for our abstract theorem to apply.

The technical bulk of the paper is devoted to a detailed treatment of the lowerhedging and the utility-maximization problems of financial mathematics. It is in these - but certainly not exclusively in these - moderately nonstandard control problems where the power of our approach seems to be evident. The first example we focus on deals with the problem of lower hedging (sub-replication) in rather general financial markets. We showcase the power of our framework by establishing a DPP for this problem when the stock prices, along with non-traded factor processes, form a RCLL Feller process with a Hausdorff LCCB state space. Additional conditions imposed on the model are minimal: the classical no-arbitrage (NFLVR) assumption and the weak requirement of local boundedness from below (which is, e.g., implied by continuity or nonnegativity of the stock price). Under these conditions, the collections of equivalent local-martingale measures - parametrized by the initial condition - form a controlled Markov family and, additionally, satisfy the condition of disintegrability.

The results obtained for lower hedging are further developed to show that the DPP holds for the utility-maximization problem with a random endowment under the same, minimal, regularity assumptions. The way this problem is tackled sheds additional light on how our framework may be used: unlike in the lower hedging problem, the set of equivalent local martingale measures itself does not play the role of the controlled Markov family, but it parametrizes it in a Borel-measurable way. Thanks to good stability properties of analytic sets, this is enough to guarantee the assumptions of the abstract theorem and, consequently, imply the DPP for this problem, too.

1.4. The structure of the paper. After this introduction, section 2 outlines the abstract setup and presents our main abstract theorem together with its proof. First examples and a necessary condition for the assumptions of the theory to hold are also given. Section 3 applies the abstract results to the problems of lower hedging and utility maximization in financial mathematics. A short appendix contains a telegraphic primer (in place primarily to fix the terminology) on the pertinent concepts and results of basic descriptive set theory. 
2. An abstract framework for the dynamic programming principle. We start by describing a fairly general probabilistic setting for the weak formulation of optimal stochastic control. The reader will observe that - in its essence, at least - it is a marriage between the classical discrete-time framework as described, for example, in [6], and the standard set-up for Markov families on the canonical space of rightcontinuous paths with left limits, as outlined, for instance, in [25]. In fact, an effort has been made to stress the formal analogy with the abstract structure of a Markov family as much as possible. We remind the reader that a short glossary of necessary descriptive-set-theoretic terms (used below) is given in the appendix.

2.1. Elements of the setup. For completeness and definiteness, we start by defining some standard elements of the setup.

The space $D_{E}$. Given a Polish space $E$ (a topological space homeomorphic to a complete separable metric space), let $D_{E}$ be the set of all $E$-valued RCLL paths on $[0, \infty)$. A generic path in $D_{E}$ is typically denoted by $\omega$ and the map $X_{t}: D_{E} \rightarrow E$, defined for $t \in[0, \infty)$ and $\omega \in D_{E}$ by $X_{t}(\omega)=\omega(t)$, is referred to as the coordinate mapping. It is always assumed that an isolated point $\iota$ is adjoined to $E$ (in addition to any "cemetery"-type points already present to deal with "killing" and sub-probability measures). This way, the index set for the coordinate mappings can be naturally extended to the class of all maps $\tau: D_{E} \rightarrow[0, \infty]$ by setting $X_{\tau}(\omega)=X_{\tau(\omega)}(\omega)$, when $\tau(\omega)<\infty$; and $X_{\tau}(\omega)=\iota$, otherwise.

The family $\left(\theta_{t}\right)_{t \geq 0}$ of shift operators is defined on $D_{E}$ by $X_{s}\left(\theta_{t}(\omega)\right)=X_{t+s}(\omega)$, for all $s, t \geq 0$ and $\omega \in D_{E}$. Just like in the case of the coordinate mappings, definitions of shift operators can be extended to include random-time-valued indices, namely by setting $X_{t}\left(\theta_{\tau}(\omega)\right)=X_{\tau(\omega)+t}(\omega)$; here $\theta_{\tau}(\omega)=\omega_{\iota}$ when $\tau(\omega)=+\infty$ with $\omega_{\iota}$ denoting the constant trajectory with the value $\iota$. Informally, unless otherwise stated, we imagine all paths as taking the constant value $\iota$ "at" and "after" $+\infty$.

The space $D_{E}$ is always assumed to be equipped with the Skorokhod topology; this way it inherits the structure of a Polish space itself (see [25, Theorem 5.6, p. 121]). While quite important for the Polish property of $D_{E}$, the choice of the Skorokhod topology is not crucial as far as the Borel $\sigma$-algebra $\mathcal{B}\left(D_{E}\right)$, generated by it on $D_{E}$, is concerned. Indeed, it can be shown that it is generated by most other, often used, metrics on $D_{E}$; in fact, $\mathcal{B}\left(D_{E}\right)$ is simply the $\sigma$-algebra generated by the coordinate maps (see [25, Proposition 7.1, p. 127]).

Probability measures on $D_{E}$. The set of all probability measures on $D_{E}$ is denoted by $\mathfrak{P}\left(D_{E}\right)$ or, simply, by $\mathfrak{P}$, if no confusion is anticipated. Since we only consider the Borel $\sigma$-algebra $\mathcal{B}\left(D_{E}\right)$ on $D_{E}$, we permit ourselves to abuse the language in the usual way, and refer to $\mu \in \mathfrak{P}$ as a "probability on $D_{E}$ ", as opposed to "probability on $\mathcal{B}\left(D_{E}\right)$ ". As usual, $\mathfrak{P}$ is endowed with the topology of weak convergence, which gives it the structure of a Polish space (see [25, Theorem 1.7, p. 101]) and generates the Borel $\sigma$-algebra $\mathcal{B}(\mathfrak{P})$.

We simplify the exposition by adopting some of the probabilistic terminology and notation. For example, depending on the context, we use either the probabilistic $\mathbb{E}^{\mu}[G]$ or the analytic $\int G d \mu$ (or $\int G(\omega) \mu(d \omega)$ ) notation to denote the integral of the appropriately measurable function $G$ with respect to the probability measure $\mu$ over 
$D_{E}$. In general, the measurable space $\left(D_{E}, \mathcal{B}\left(D_{E}\right)\right)$, together with some probability measure $\mu \in \mathfrak{P}$, will play the role of the underlying probability space for the remainder of the paper; when probabilistic terminology is used, it will be with respect to this space.

Universally measurable kernels and random variables. A map $\nu: E \times \mathcal{B}\left(D_{E}\right) \rightarrow$ $[0,1]$ is called a universally measurable kernel (or, simply, kernel) if

1. $\nu(x, \cdot) \in \mathfrak{P}$ for all $x \in E$, and

2. $E \ni x \mapsto \nu(x, A)$ is universally measurable for all $A \in \mathcal{B}\left(D_{E}\right)$.

Due to the special nature of the state $\iota$, we always assume that $\nu_{\iota}(A)=\mathbf{1}_{\left\{\omega_{\iota} \in A\right\}}$, i.e., that $\nu_{\iota}$ is necessarily the Dirac mass at the constant trajectory $\omega_{\iota}$.

To make the notation easier to read, we often write $\nu_{x}$ for the probability measure $\nu(x, \cdot)$ and interpret $\nu$ as a map $E \rightarrow \mathfrak{P}$. A classical result of Varadarajan (see [43, Lemma 2.3., p. 194]) states that a universally measurable kernel, when interpreted this way, defines a universally-measurable map $E \rightarrow \mathfrak{P}$. Since $\mathfrak{P}$ is Polish, the graph $\Gamma_{\nu}$ of $\nu$, given by $\Gamma_{\nu}=\left\{\left(x, \nu_{x}\right): x \in E\right\} \subseteq E \times \mathfrak{P}$ is a product-measurable subset of $E \times \mathfrak{P}$, when $E$ is equipped with the universally-measurable $\sigma$-algebra.

In the spirit of our interpretation of $\left(D_{E}, \mathcal{B}\left(D_{E}\right)\right)$ as a probability space, a universally-measurable map $G: D_{E} \rightarrow[-\infty, \infty]$ is called a universally measurable random variable (or, simply, random variable). Unless otherwise specified, we always set $G(\iota)=0$.

Given a family $\mathfrak{R} \subset \mathfrak{P}$ of probability measures on $D_{E}$, a random variable $G$ is said to be $\mathfrak{R}$-lower semi-integrable if $\mathbb{E}^{\mu}\left[G^{-}\right]<\infty$, for all $\mu \in \mathfrak{R}$; the set of all $\mathfrak{R}$-lower semi-integrable random variables is denoted by $\mathcal{L}^{0-1}(\mathfrak{R})$. For $\mu \in \mathfrak{P}$ and $G \in \mathcal{L}^{0-1}(\{\mu\})$, the expectation $\mathbb{E}^{\mu}[G]$ is well-defined, with values in $(-\infty, \infty]$ by setting $\mathbb{E}^{\mu}[G]=\mathbb{E}^{\mu}\left[G^{+}\right]-\mathbb{E}^{\mu}\left[G^{-}\right]$. We note that, given a kernel $\nu$ and a random variable $G \in \mathcal{L}^{0-1}\left(\left\{\nu_{x}\right\}_{x \in E}\right)$, the integral $g(x)=\int G(\omega) \nu_{x}(d \omega)$ defines a universally measurable map $g: E \rightarrow(-\infty, \infty]$.

Concatenation of paths. A Borel-measurable map from $D_{E}$ to $[0, \infty]$ is called a random time. For a random time $\tau$ and two paths $\omega, \omega^{\prime} \in D_{E}$, the concatenation $\omega *_{\tau} \omega^{\prime}$ of $\omega$ and $\omega^{\prime}$ at $\tau$ is an element of $D_{E}$ whose value at $t \geq 0$ is given by

$$
X_{t}\left(\omega *_{\tau} \omega^{\prime}\right)= \begin{cases}X_{t}(\omega), & t<\tau(\omega) \\ X_{\tau}(\omega)+X_{t-\tau(\omega)}\left(\omega^{\prime}\right)-X_{0}\left(\omega^{\prime}\right), & t \geq \tau(\omega)\end{cases}
$$

It follows immediately that for $\omega, \omega^{\prime} \in D_{E}$ and a random time $\tau$, we have

$$
\omega *_{\tau} \theta_{\tau}(\omega)=\omega, \text { and } \theta_{\tau}\left(\omega *_{\tau} \omega^{\prime}\right)= \begin{cases}\omega^{\prime}+\left(X_{\tau}(\omega)-X_{0}\left(\omega^{\prime}\right)\right), & \tau(\omega)<\infty \\ \omega_{\iota}, & \tau(\omega)=\infty .\end{cases}
$$

The map $\left(\omega, \omega^{\prime}\right) \mapsto X_{t}\left(\omega *_{\tau} \omega^{\prime}\right)$ is easily seen to be $\left(\mathcal{B}\left(D_{E}\right) \otimes \mathcal{B}\left(D_{E}\right), \mathcal{B}(E)\right)$-measurable for each $t \geq 0$. Thanks to the fact that the Borel $\sigma$-algebra $\mathcal{B}\left(D_{E}\right)$ on $D_{E}$ is generated by the family of coordinate maps, the concatenation map $D_{E} \times D_{E} \rightarrow D_{E}$ is Borelmeasurable, as well. 
Concatenation of laws. For a random time $\tau$, a probability measure $\mu \in \mathfrak{P}$ and a kernel $\nu$ we define the product $\mu \otimes_{\tau} \nu$ as the probability measure on $D_{E} \times D_{E}$ with

$$
\left(\mu \otimes_{\tau} \nu\right)[B]=\iint \mathbf{1}_{B}\left(\omega, \omega^{\prime}\right) \nu_{X_{\tau}(\omega)}\left(d \omega^{\prime}\right) \mu(d \omega), \text { for } B \in \mathcal{B}\left(D_{E} \times D_{E}\right) .
$$

The concatenation $\mu *_{\tau} \nu$ of $\mu$ and $\nu$ at $\tau$ is the probability measure $\mu *_{\tau} \nu$ on $D_{E}$, given by

$$
\left(\mu *_{\tau} \nu\right)[A]=\iint \mathbf{1}_{A}\left(\omega *_{\tau} \omega^{\prime}\right) \mu \otimes_{\tau} \nu\left(d \omega, d \omega^{\prime}\right), \text { for } A \in \mathcal{B}\left(D_{E}\right) .
$$

We observe that the fact that $X_{\tau}=\iota$ on $\{\tau=\infty\}$ plays a minimal role in the definition (2.3) of $\mu *_{\tau} \nu$ above. Indeed, if $\tau(\omega)=\infty$, we have $\omega *_{\tau} \omega^{\prime}=\omega$, and the inner integral is applied to the constant function $\omega^{\prime} \mapsto \mathbf{1}_{A}(\omega)$. Therefore, its value is simply $\mathbf{1}_{A}(\omega)$ as soon as $\nu_{X_{\tau}}$ is (an arbitrary) probability measure. Accordingly, for a sufficiently integrable (nonnegative, for example) random variable $G$, we have

$$
\mathbb{E}^{\mu *_{\tau} \nu}[G]=\mathbb{E}^{\mu}[\tilde{G}], \text { where } \tilde{G}(\omega)= \begin{cases}\mathbb{E}^{\nu_{X_{\tau}(\omega)}}\left[G\left(\omega *_{\tau} \cdot\right)\right], & \tau(\omega)<\infty \\ G(\omega), & \tau(\omega)=\infty\end{cases}
$$

In particular, we note that for $G \in \mathcal{L}^{0-1}(\{\mu \otimes \nu: \mu \in \mathfrak{R}\})$, we have $\tilde{G} \in \mathcal{L}^{0-1}(\mathfrak{R})$, for any collection $\mathfrak{R} \subseteq \mathfrak{P}$.

Controlled Markov families. Let $\mathcal{P}=\left(\mathcal{P}^{x}\right)_{x \in E}$ be a family of non-empty subsets of $\mathfrak{P}$. A universally measurable kernel $\nu$ with $\nu_{x} \in \mathcal{P}^{x}$, for all $x \in E$, is called a $\mathcal{P}$-selector; the set of all $\mathcal{P}$-selectors is denoted by $\mathcal{S}(\mathcal{P})$. We note that a kernel $\nu$ is a $\mathcal{P}$-selector if and only if $\Gamma_{\nu} \subseteq \Gamma_{\mathcal{P}}$, where $\Gamma_{\mathcal{P}}=\left\{(x, \mu): \mu \in \mathcal{P}^{x}\right\} \subseteq E \times \mathfrak{P}$.

Definition 2.1. A pair $(\mathcal{P}, \mathcal{T})$, consisting of a family $\mathcal{P}=\left(\mathcal{P}^{x}\right)_{x \in E}$ of nonempty subsets of $\mathfrak{P}$ and a set $\mathcal{T}$ of random times is called a controlled Markov family if

1. the graph $\Gamma_{\mathcal{P}}=\left\{(x, \mu): \mu \in \mathcal{P}^{x}\right\}$ is an analytic subset of $E \times \mathfrak{P}$, and

2. for $x \in E, \mu \in \mathcal{P}^{x}, \tau \in \mathcal{T}$ and $\nu \in \mathcal{S}(\mathcal{P})$, we have $\mu *_{\tau} \nu \in \mathcal{P}^{x}$.

We refer to the first property above as analyticity and to the second one as concatenability.

We think of a controlled Markov family as a stripped-down version of a mechanism by which a stochastic system is controlled; a measure $\mu \in \mathcal{P}^{x}$, chosen by the controller in the initial state $x \in E$, serves as the probability law of the system's future evolution. Closedness under concatenation - a proxy for the Chapman-Kolmogorov relations models the controller's freedom to switch to a different control, within the admissible class, at any point $\tau \in \mathcal{T}$. The set $\mathcal{T}$ of random times typically forms either the family of all deterministic times, or the family of all stopping times, corresponding, respectively, to Markov and strong Markov families. Other choices, such as the set of optional times, stopping times satisfying certain integrability constraints, etc., could also be of interest. 
Markov control problems and their value functions. A triplet $(\mathcal{P}, \mathcal{T}, G)$, consisting of a controlled Markov family $(\mathcal{P}, \mathcal{T})$ and a random variable $G \in \mathcal{L}^{0-1}(\mathcal{P})=$ $\mathcal{L}^{0-1}\left(\cup_{x} \mathcal{P}^{x}\right)$ is called a Markov control problem. Its value function $v: E \rightarrow$ $[-\infty, \infty]$ is defined by

$$
v(x)=\inf _{\mu \in \mathcal{P}^{x}} \mathbb{E}^{\mu}[G], \text { for } x \in E .
$$

Given $\varepsilon>0$, a probability measure $\mu \in \mathcal{P}^{x}$ is said to be $\varepsilon$-optimal if $\mathbb{E}^{\mu}[G]<v(x)+\varepsilon$, with the usual convention that $-\infty+\varepsilon=-1 / \varepsilon$.

A Markov control problem is simply a controlled Markov family together with a minimally integrable random variable $G$, which we interpret as the cost associated with a state of the world. The controller's objective - quantified by the value function $v$ - is to minimize the expected value of this cost.

Tail random variables and (approximate) disintegrability. We give here a general definition of a class of random variables which posess a mild shift-invariance property.

Definition 2.2. Given a controlled Markov family $(\mathcal{P}, \mathcal{T})$, a Borel-measurable random variable $G \in \mathcal{L}^{0-1}(\mathcal{P})$ is said to be a $(\mathcal{T}, \mathcal{P})$-tail random variable if, for all $(x, \mu) \in \Gamma_{\mathcal{P}}, \nu \in \mathcal{S}(\mathcal{P})$ and $\tau \in \mathcal{T}$ we have

$$
\mathbb{E}^{\mu *_{\tau} \nu}[G]=\mathbb{E}^{\mu}\left[g\left(X_{\tau}\right) \mathbf{1}_{\{\tau<\infty\}}+G \mathbf{1}_{\{\tau=\infty\}}\right] \text {, where } g(x)=\mathbb{E}^{\nu_{x}}[G] .
$$

Tail random variables can be loosely interpreted as those that depend only on the future after each $\tau \in \mathcal{T}$. They will be used as cost functionals for our main control problem, as defined below. One could argue that only tail random variables matter, as far as Markov control problems are concerned, since there is little point in applying different controls to a system whose final cost is already known. It will be shown in Proposition 2.5 below that, under mild additional assumptions, a random variable $G$ with $G \circ \theta_{t}=G$, for all $t$, is a tail random variable.

Definition 2.3. Given a controlled Markov family $(\mathcal{P}, \mathcal{T})$, we say that a random variable $G$ is $(\mathcal{P}, \mathcal{T})$-approximately disintegrable if $G$ is a $(\mathcal{P}, \mathcal{T})$-tail random variable and

$$
\forall(x, \mu) \in \Gamma_{\mathcal{P}}, \tau \in \mathcal{T}, \varepsilon>0, \quad \exists \nu \in \mathcal{S}(\mathcal{P}), \quad \mathbb{E}^{\mu *_{\tau} \nu}[G] \leq \mathbb{E}^{\mu}[G]+\varepsilon .
$$

$G$ is called $(\mathcal{P}, \mathcal{T})$-disintegrable if (2.7) holds for $\varepsilon=0$, as well.

The notion of (approximate) disintegrability is, in a sense, dual to that of closedness under concatenation of Definition 2.1. If a family, closed under concatenation, allows the controller to change his/her mind at any point, (approximate) disintegrability states that the controller can remain (near) optimal if forced to do so. We also note, for future use, that approximate disintegrability allows us to construct $\varepsilon$-optimizers of the form $\mu *_{\tau} \nu$, for $\mu \in \mathcal{P}^{x}, \nu \in \mathcal{S}(\mathcal{P})$ and $\tau \in \mathcal{T}$. 
2.2. An abstract dynamic-programming principle. With the notions of a Markov control problem, (approximate) disintegrability and the value function introduce above, we are now ready to state our abstract version of the DPP. The reader familiar with the discrete-time theory will observe that, once the framework has been set up, the proof is quite standard and, for the most part, follows the well-known steps.

Theorem 2.4 (An Abstract dynamic programming Principle). Let $(\mathcal{P}, \mathcal{T}, G)$ be a Markov control problem with $G(\mathcal{P}, \mathcal{T})$-approximately disintegrable, and let $v$ be its value function. Then:

1. The function $v$ is lower semi-analytic and, hence, universally measurable.

2. For each $\varepsilon>0$ there exists a universally-measurable selector $\hat{\nu}^{\varepsilon} \in \mathcal{S}(\mathcal{P})$ such that $\hat{\nu}_{x}^{\varepsilon}$ is $\varepsilon$-optimal for each $x \in E$.

3. The dynamic-programming principle (DPP) holds at all $\tau \in \mathcal{T}$; more precisely, following the convention that that $\mathbb{E}^{\mu}[Y]=\infty$ as soon as $\mathbb{E}^{\mu}\left[Y^{+}\right]=\infty$, we have

$$
v(x)=\inf _{\mu \in \mathcal{P}^{x}} \mathbb{E}^{\mu}\left[v\left(X_{\tau}\right) \mathbf{1}_{\{\tau<\infty\}}+G \mathbf{1}_{\{\tau=\infty\}}\right] \text { for all } x \in E \text { and } \tau \in \mathcal{T},
$$

Proof.

1. The statement clearly holds if $v(x)=+\infty$, for all $x \in E$. Otherwise, for $a \in\left(\inf _{x \in E} v(x), \infty\right)$, we construct the sub-level set

$$
\{x \in E: v(x)<a\}=\left\{x \in E: \mathbb{E}^{\mu}[G]<a \text { for some } \mu \in \mathcal{P}^{x}\right\}=\operatorname{proj}_{E} J^{<a},
$$

where $\operatorname{proj}_{E}$ denotes the natural projection from $E \times \mathfrak{P}$ onto $E$, and

$$
J^{<a}=\left\{(x, \mu): \mathbb{E}^{\mu}\left[G^{+}\right]<a+\mathbb{E}^{\mu}\left[G^{-}\right]\right\} \bigcap\left\{(x, \mu): \mu \in \mathcal{P}^{x}\right\} \subseteq E \times \mathfrak{P} .
$$

Since the maps $\mu \mapsto \mathbb{E}^{\mu}\left[G^{+}\right]$and $\mu \mapsto \mathbb{E}^{\mu}\left[G^{-}\right]$are Borel measurable and graph $\Gamma_{\mathcal{P}}=\left\{(x, \mu): \mu \in \mathcal{P}^{x}\right\}$ is analytic, both sets in the definition of $J^{<a}$ are analytic. Therefore, so are the set $J^{<a}$ and its projection $\{v<a\}$. Consequently, $v$ is lower semi-analytic and, a fortiori, universally measurable.

2. Let the set $\Gamma_{\mathcal{P}_{\varepsilon}}$ be given by

$$
\Gamma_{\mathcal{P}_{\varepsilon}}=\left\{(x, \mu) \in E \times \mathfrak{P}: \mathbb{E}^{\mu}\left[G^{+}\right] \leq \mathbb{E}^{\mu}\left[G^{-}\right]+(v(x)+\varepsilon)\right\} \bigcap \Gamma_{\mathcal{P}},
$$

where the convention $-\infty+\varepsilon=-1 / \varepsilon$ is used for $v(x)+\varepsilon$. Since $v$ is universally measurable, $\Gamma_{\mathcal{P}_{\varepsilon}}$ is $\mathcal{U} \times \mathcal{B}(\mathfrak{P})$-measurable. Thanks to the fact that the universal $\sigma$ algebra is closed under the Suslin operation ([39, Theorem 3.5.22., p. 114]), we can apply (a slight generalization of) the Jankov-von Neumann theorem ([39, Theorem 5.7 .5$, p. 200]) and conclude that a "universally-measurable section", i.e., a kernel $\hat{\nu}^{\varepsilon}$ with $\Gamma_{\hat{\nu}^{\varepsilon}} \subseteq \Gamma_{\mathcal{P}^{\varepsilon}}$ exists. Therefore, $\hat{\nu}^{\varepsilon} \in \mathcal{S}(\mathcal{P})$, and, clearly, $\hat{\nu}_{x}^{\varepsilon}$ is $\varepsilon$-optimal for each $x \in E$.

3. To simplify the notation, we use the convention $g\left(X_{\tau}\right)=v\left(X_{\tau}\right)=G$ on $\{\tau=$ $\infty$ \}. Given $x \in E, \tau \in \mathcal{T}$ and $\varepsilon>0$, the assumption of approximate disintegrability allows us to choose an $\varepsilon$-optimal measure of the form $\hat{\mu} *_{\tau} \hat{\nu}$, for some $\hat{\mu} \in \mathcal{P}^{x}$ and $\hat{\nu} \in$ 
$\mathcal{S}(\mathcal{P})$. The universally-measurable map $g: E \rightarrow(-\infty, \infty]$, defined by $g(x)=\mathbb{E}^{\hat{\nu}_{x}}[G]$ for $x \in E$ satisfies $g(x) \geq v(x)$, for all $x \in E$. On the other hand, the tail property of $G$, as in (2.6), yields

$$
v(x)+\varepsilon>\mathbb{E}^{\hat{\mu} *_{\tau} \hat{\nu}}[G]=\mathbb{E}^{\hat{\mu}}\left[g\left(X_{\tau}\right)\right] .
$$

Without loss of generality we assume that $v(x)<\infty$, so that for large enough $n \in \mathbb{N}$,

$$
v(x)+\varepsilon>\mathbb{E}^{\hat{\mu}}\left[\max \left(-n, g\left(X_{\tau}\right)\right)\right] \geq \mathbb{E}^{\hat{\mu}}\left[\max \left(-n, v\left(X_{\tau}\right)\right] .\right.
$$

Consequently, with the convention $v\left(X_{\tau}\right)=G$ on $\{\tau=\infty\}$, we have

$$
v(x) \geq \inf _{\mu \in \mathcal{P}^{x}} \inf _{n \in \mathbb{N}} \mathbb{E}^{\mu}\left[\max \left(-n, v\left(X_{\tau}\right)\right)\right], \text { for all } x \in E .
$$

For the opposite inequality, using (2) above, for each $\varepsilon>0$ we can pick a kernel $\hat{\nu}^{\varepsilon} \in \mathcal{S}(\mathcal{P})$ such that $\hat{\nu}_{x}^{\varepsilon}$ is $\varepsilon$-optimal for each $x \in E$, and set $g(x)=\mathbb{E}^{\hat{\nu}_{x}^{\varepsilon}}[G]$ so that $g$ is universally measurable and $g(x) \leq v(x)+\varepsilon$, for all $x \in E$. For $x_{0} \in E$ with $v\left(x_{0}\right)>-\infty$, an arbitrary $\mu \in \mathcal{P}^{x_{0}}$, and $n \in \mathbb{N}$, we have

$$
\begin{aligned}
v\left(x_{0}\right) & \leq \mathbb{E}^{\mu *_{\tau} \hat{\nu}^{\varepsilon}}[G]=\mathbb{E}^{\mu}\left[g\left(X_{\tau}\right)\right] \leq \mathbb{E}^{\mu}\left[\max \left(-n, g\left(X_{\tau}\right)\right)\right] \\
& \leq \mathbb{E}^{\mu}\left[\max \left(-n, v\left(X_{\tau}\right)+\varepsilon\right)\right] .
\end{aligned}
$$

Assuming, without loss of generality, that $\mathbb{E}^{\mu}\left[v\left(X_{\tau}\right)^{+}\right]<\infty$, we use the Dominated convergence theorem to conclude that

$$
v\left(x_{0}\right) \leq \mathbb{E}^{\mu}\left[\max \left(-n, v\left(X_{\tau}\right)\right)\right] .
$$

To complete the proof, we take the infimum over all $\mu \in \mathcal{P}^{x_{0}}$ and all $n \in \mathbb{N}$.

The analyticity assumption of Definition 2.1 of a controlled Markov family plays a major role in the proof of the parts (1) and (2) of Theorem 2.4. In contrast, closedness under concatenation and the (approximate) disintegrability are only used in part (3), one for each of the two opposite inequalities which consitute (2.8).

2.3. A sufficient condition for disintegrability. Next, we present a simple sufficient condition for disintegrability and the tail property, applicable in most cases of interest. For $x \in E$, a random time $\tau$ and a probability measure $\mu$ on $D_{E}$, we let

$$
(\xi, B) \mapsto \mu\left[\theta_{\tau} \in B \mid X_{\tau}=\xi\right], \quad(\xi, B) \in E \times \mathcal{B}\left(D_{E}\right),
$$

denote a regular version of the conditional distribution of $\theta_{\tau}$, given $X_{\tau}$, under $\mu$; its existence - as a map defined $\mu \circ X_{\tau}^{-1}$-a.s. - is guaranteed by the Polish property of $D_{E}$ (see, e.g., Theorem 5.3, p. 84., in [29]). We remind the reader that $X_{\tau}=\iota$ on $\tau=\infty$, and that, under any $\mu$, by convention, $\iota$ is absorbing, i.e., that $\mu\left[\cdot \mid X_{\tau}=\iota\right]$ is the Dirac mass on $\omega_{\iota}$ when $\mu\left[X_{\tau}=\iota\right]>0$.

Proposition 2.5 (A sufficient condition for disintegrability). Let $(\mathcal{P}, \mathcal{T})$ be a controlled Markov family, and let $G$ be a Borel random variable in $\mathcal{L}^{0-1}(\mathcal{P})$ such that

$$
G\left(\theta_{t}(\omega)\right)=G(\omega) \text { for all } t \geq 0, \omega \in D_{E} .
$$


Then $G$ is a $(\mathcal{P}, \mathcal{T})$-tail random variable.

If, in addition, for each $\mu \in \cup_{x} \mathcal{P}^{x}$ and $\tau \in \mathcal{T}$ we have

$$
\mu\left[\theta_{\tau} \in \cdot \mid X_{\tau}=x\right] \in \mathcal{P}^{x}, \text { for } \mu \circ X_{\tau}^{-1} \text {-almost all } x \in E,
$$

then $G$ is $(\mathcal{P}, \mathcal{T})$-disintegrable.

Proof. First, we prove that a random variable $G \in \mathcal{L}^{0-1}(\mathcal{P})$, which satisfies $(2.10)$, is a tail random variable. The relations in (2.1) imply that for all $\omega, \omega^{\prime} \in D_{E}$, and any random time $\tau$, we have $G\left(\omega *_{\tau} \omega^{\prime}\right)=G\left(\omega^{\prime}\right)$ as soon as $\tau(\omega)<\infty$ and $X_{\tau}(\omega)=X_{0}\left(\omega^{\prime}\right)$. Therefore, for $(x, \mu) \in \Gamma_{\mathcal{P}}, \tau \in \mathcal{T}, \nu \in \mathcal{S}(\mathcal{P})$, and $\omega \in D_{E}$ such that $\tau(\omega)<\infty$, integration against $\nu_{X_{\tau}(\omega)}$ yields

$$
\int G\left(\omega *_{\tau} \omega^{\prime}\right) \nu_{X_{\tau}(\omega)}\left(d \omega^{\prime}\right)=g\left(X_{\tau}(\omega)\right), \text { where } g(x)=\int G\left(\omega^{\prime}\right) \nu_{x}\left(d \omega^{\prime}\right) .
$$

When $\tau(\omega)=\infty$, we have $\int G\left(\omega *_{\tau} \omega^{\prime}\right) \nu_{X_{\tau}(\omega)}\left(d \omega^{\prime}\right)=G(\omega)$, and so

$$
\mathbb{E}^{\mu *_{\tau} \nu}[G]=\iint G\left(\omega *_{\tau} \omega^{\prime}\right) \nu_{X_{\tau}(\omega)}\left(d \omega^{\prime}\right) \mu(d \omega)=\mathbb{E}^{\mu}\left[g\left(X_{\tau}\right) \mathbf{1}_{\{\tau<\infty\}}+G \mathbf{1}_{\{\tau=\infty\}}\right] .
$$

Next, we focus on disintegrability, and fix $\mu \in \cup_{x} \mathcal{P}^{x}$. The condition (2.11) requires that the regular version of $\mu\left[\theta_{\tau} \in \cdot \mid X_{\tau}=x\right]$ belongs to $\mathcal{P}^{x}$, but only for $\mu \circ X_{\tau}^{-1}$ almost all $x$. We can, however, easily redefine it on a Borel subset $F$ of $D_{E}$, while keeping measurability in the first variable. Moreover, thanks to the nonemptyness of $\mathcal{S}(\mathcal{P})$, we can arrange the redefinition so that the newly obtained version is a kernel in $\mathcal{S}(\mathcal{P})$. We denote this new version by $\mu_{x}^{\tau}$, with the usual interpretation that $\mu_{x}^{\tau}[B]=\mu\left[\theta_{\tau} \in B \mid X_{\tau}=x\right]$.

The definition of the regular conditional distribution implies that

$$
\mathbb{E}^{\mu}\left[G \circ \theta_{\tau}\right]=\iint G\left(\omega^{\prime}\right) \mu_{X_{\tau}(\omega)}^{\tau}\left(d \omega^{\prime}\right) \mu(d \omega),
$$

so that, using the fact (implied by (2.10)) that $G\left(\omega^{\prime}\right) \mathbf{1}_{\{\tau(\omega)<\infty\}}=G\left(\omega *_{\tau} \omega^{\prime}\right) \mathbf{1}_{\{\tau(\omega)<\infty\}}$, for all $\omega, \omega^{\prime} \in D_{E}$ with $X_{\tau}(\omega)=X_{0}\left(\omega^{\prime}\right)$ and $\tau(\omega)<\infty$, we have

$$
\begin{aligned}
\mathbb{E}^{\mu}\left[G \circ \theta_{\tau}\right] & +\iint G\left(\omega *_{\tau} \omega^{\prime}\right) \mathbf{1}_{\{\tau(\omega)=\infty\}} \mu_{X_{\tau}(\omega)}^{\tau}\left(d \omega^{\prime}\right) \mu(d \omega) \\
& =\iint\left(G\left(\omega *_{\tau} \omega^{\prime}\right)+G\left(\omega^{\prime}\right) \mathbf{1}_{\{\tau(\omega)=\infty\}}\right) \mu_{X_{\tau}(\omega)}^{\tau}\left(d \omega^{\prime}\right) \mu(d \omega)=\mathbb{E}^{\mu *_{\tau} \nu}[G]
\end{aligned}
$$

where the convention that $G\left(\omega_{\iota}\right)=0$ is used. On the other hand, that same convention, and the assumption (2.10) yield that $G \circ \theta_{\tau}=G \mathbf{1}_{\{\tau<\infty\}}$ and

$$
\iint G\left(\omega *_{\tau} \omega^{\prime}\right) \mathbf{1}_{\{\tau(\omega)=\infty\}} \mu_{X_{\tau}(\omega)}^{\tau}\left(d \omega^{\prime}\right) \mu(d \omega)=\mathbb{E}^{\mu}\left[G \mathbf{1}_{\{\tau=\infty\}}\right] .
$$

A typical case in which (2.10) in Proposition 2.5 holds is when $G$ is of the form $G(\omega)=\lim _{t \rightarrow \infty} \gamma\left(X_{t}(\omega)\right)$, for some Borel function $\gamma: E \rightarrow \mathbb{R}$, where the limit above should be interpreted in an appropriate sense (see Lemma 3.12 for a precise 
description). In most applications, it exists as an a.s.-limit with respect to all $\mu \in$ $\cup_{x} \mathcal{P}^{x}$. Almost all stochastic control problems of interest, such as those with the finite time horizon, running cost, or, more generally, cost upon absorption in finite or infinite time can easily be seen to fall under its domain.

As for (2.11), it simply states that for each control $\mu$ and each stopping time $\tau \in \mathcal{T}$, the controller can switch to a currently available control is such a way that that system continues to evolve as if no switch has been made at all. In particular, if forced to make a switch, the controller can do it in such a way that the expected cost stays exactly the same.

2.4. First examples. To familiarize the reader with the content and scope of Definition 2.3, we present several general examples in various degrees of detail; in most cases, the full treatment is outside the scope of this paper.

(Strong) Markov families. In a prototypical example of a controlled Markov family all the sets $\mathcal{P}^{x}$ are singletons. Indeed, for a (strong) Markov family $\left(\mathbb{P}^{x}\right)_{x \in E}$ on the canonical space $D_{E}$, one can easily show that the assignment $\mathcal{P}^{x}=\left\{\mathbb{P}^{x}\right\}$ for $x \in E$ defines a controlled Markov family in the sense of Definition 2.3. This can be achieved, for example, if $\left(\mathbb{P}^{x}\right)_{x \in E}$ has the Feller property, but the (strong) Markov property and the RCLL paths will suffice. In that case, $\mathcal{P}^{x}$, together with the set $\mathcal{T}$ of all optional times for the canonical filtration - or just deterministic times if the Markov property is not strong - and the appropriate tail random variable $G$ form a controlled Markov family. While the concatenation and disintegration properties are simple reformulations of the Chapman-Kolmogorov equations and the (strong) Markov property (via Proposition 2.5), the analyticity of the graph $\Gamma_{\mathcal{P}}$ follows from Borel-measurability of the map $x \mapsto \mathbb{P}^{x}$. Indeed, the Markov property implies that (see [25, Proposition 1.2, p. 158]) the map $x \mapsto \mathbb{P}^{x}[B]$ is Borel-measurable for each $B \in \mathcal{B}\left(D_{E}\right)$. A classical result of Varadarajan (see [43, Lemma 2.3., p. 194]) states that this weak form of measurability (sometimes referred to as customary Borel measurability) implies that the map $x \mapsto \mathbb{P}^{x}$ (with the co-domain $\mathfrak{P}$ ) - and, therefore, its graph - is Borel measurable, and, a fortiori, analytic.

The conclusions of our main result (Theorem 2.4) are not novel or particularly illuminating in this case. The lower semianalyticity in (1) can be strengthened to Borel measurability and the existence of a universally measurable selector in (2) is trivially verified. The third conclusion simply reformulates the (strong) Markov property, stating that the function of the form $v(x)=\mathbb{E}^{x}[G]$ has the mean-value property. The true significance of this example is to aid intuition by drawing parallels between the notion of a controlled Markov family and its stencil, a Markov family of probability measures on $\mathcal{B}\left(D_{E}\right)$.

Multiple solutions to the martingale problem. One of the most versatile and studied methods of constructing Feller families on $\mathcal{B}\left(D_{E}\right)$ is through the martingale problem of Stroock and Varadhan. Under appropriate regularity conditions (which we skip here and refer the reader to [25] or [42] for details), one picks a (typically local or nonlocal differential) operator $A$ defined on a class $\mathcal{A}$ of functions $f: E \rightarrow \mathbb{R}$ and constructs a family of families of measures $\left(\mathcal{P}^{x}\right)_{x \in E}$ on $D_{E}$ such that

1. $\mu\left(X_{0}=x\right)=1$, for all $(x, \mu) \in \Gamma_{\mathcal{P}}$, 
2. under $\mu$, the process $f\left(X_{t}\right)-\int_{0}^{t} A f\left(X_{u}\right) d u$ is an $\mathbb{F}^{X}$-martingale for each $f \in \mathcal{A}$.

When a unique such measure $\mu=\mathbb{P}^{x}$ exists for each $x \in E$, it can be shown under right regularity circumstances that the family $\left(\mathbb{P}^{x}\right)_{x \in E}$ is a Feller family and that the infinitesimal generator of the associated semigroup is $A$.

When multiple solutions of the martingale problem can be found, it is well-known that the Markov property does not necessarily hold under all of them. Very often, however, the totality of all solutions still forms a controlled Markov family in the sense of Definition 2.3. The concatenation and disintegration properties follow from the definition. The analyticity - Borel measurability, in fact - is obtained in many cases by a simple observation that $\mathcal{P}^{x}$ is defined via a countable number of Borel measurable restrictions (see, e.g., Theorem 4.2.1, p. 86 in [42], for the continuous case).

Optimal stochastic control and stopping. In the strong formulation, a typical stochastic control problem involves a set $\mathcal{N}$ of adapted (progressive, predictable, etc.) processes defined on a filtered probability space, together with a mechanism that transforms a given control $\nu$ into a stochastic process $X^{\nu}$ (typically defined on the same filtered space) and a criterion by which the performance of $X^{\nu}$ is measured. We think of $\nu$ as chosen by the controller in the effort to affect the dynamics of the state process so as to optimize an objective function, which is taken to be of the Meyer form $\mathbb{E}\left[\varphi\left(X_{T}^{\nu}\right)\right]$ on a finite horizon $[0, T]$, for the purposes of this discussion. It is immediately clear that the fundamental ingredient is not the process $\left\{X_{T}^{\nu}\right\}_{t \in[0, T]}$ itself, but its distribution, which we assume can be lifted to the space $D_{E}$, where $E$ denotes the space in which $X^{\nu}$ takes values. In other words, we view a control problem as an optimization problem over a set of probability measures on $D_{E}$, parametrized by control processes in $\mathcal{N}$.

To get a better handle on the original problem - hopefully via a dynamic programming principle - one often embeds it in a family of similar problems, parametrized by the elements of the state space (in this case $[0, T] \times E$ ). To use the abstract Theorem 2.4 , one needs the problems corresponding to various $(t, x) \in[0, T] \times E$ to fit together in a way similar to transition densities of a Markov process in a minimally measurable way. Concatenation and disintegration are typically inherited from the structure of $\mathcal{N}$ - the controls can usually be concatenated pathwise as functions, and one can usually condition away the irrelevant past values of a control $\nu \in \mathcal{N}$ to prove disintegrability. As for the analyticity of the graph $\Gamma_{\mathcal{P}}$, one needs to impose a bit of structure on $\mathcal{N}$, as well as on the distribution map, i.e., the function which translates $\nu \in \mathcal{N}$ into the distribution of $X^{\nu}$. A nice property of analyticity is that it is preserved under Borel maps; this fact allows us to deal with the case where $\mathcal{N}$ is a Borel space (a Borel subset of a Polish space) and the distribution map is merely Borel. This, in particular, covers the ubiquitous case where $\mathcal{N}$ is a Borel subset of a separable Banach space and the distribution map is defined as the solution of a controlled SDE via the martingale-problem formalism under appropriate regularity conditions. 
3. Applications to Financial Mathematics. The goal of this section is to transfer the conclusions of the abstract DPP of Theorem 2.4 to two fundamental problems of financial mathematics.

3.1. The financial market model. We start with a description of the underlying financial model common to both problems.

The state space and the Markov family of "physical" measures. We adopt the setting of section 2 with the state space $E$ of the form

$$
E=[0, \infty) \times \mathbb{R}^{d} \times F
$$

where $F$ is a locally compact Hausdorff space with a countable base (and, in particular, Polish). For notational reasons, we split the components of the coordinate process $X$ as follows

$$
X_{t}(\omega)=\left(T_{t}(\omega), S_{t}(\omega), \eta_{t}(\omega)\right)
$$

where $T, S=\left(S^{1}, S^{2}, \ldots, S^{d}\right)$ and $\eta$ take values in $[0, \infty), \mathbb{R}^{d}$ and $F$, respectively. The "physical" dynamics of $X$ will be described via a family $\left(\mathbb{P}^{x}\right)_{x \in E}$ of probability measures in $\mathfrak{P}$, i.e., on $D_{E}$, in the sense that, given the initial condition $x, \mathbb{P}^{x}$ models the evolution of the process $X$.

Let $\mathbb{F}^{0}=\left\{\mathcal{F}_{t}^{0}\right\}_{t \in[0, \infty)}$ denote the natural (raw) filtration generated by the coordinate process $X$, and let $\mathbb{F}=\left\{\mathcal{F}_{t}\right\}_{t \in[0, \infty)}$ be its right-continuous hull, i.e., $\mathcal{F}_{t}=\cap_{s>t} \mathcal{F}_{s}^{0}$, for $t \geq 0$. We postulate Markovian dynamics by enforcing the following assumption, where $\mathbb{E}^{x}$ denotes the expectation under $\mathbb{P}^{x}$ and $\mathcal{T}$ the set of all bounded $\mathbb{F}$-stopping times:

Assumption 3.1 (Markovian dynamics). The map $E \ni x \mapsto \mathbb{P}^{x} \in \mathfrak{P}$ is Borel measurable, $\mathbb{P}^{x}\left[X_{0}=x\right]=1$, for all $x \in E$, and the strong Markov property

$$
\mathbb{E}^{x}\left[Z \circ \theta_{\tau} \mid \mathcal{F}_{\tau}\right]=\mathbb{E}^{X_{\tau}}[Z], \mathbb{P}^{x} \text {-a.s. }
$$

holds for all $\tau \in \mathcal{T}, x \in E$ and all bounded Borel random variables $Z$.

A family $\left(\mathbb{P}^{x}\right)_{x \in E}$ which satisfies Assumption 3.1 above will be called a canonical strong Markov family. Such a family automatically obeys Blumenthal's 0-1 law, i.e., that, for all $t \geq 0$, we have

$$
\forall A \in \mathcal{F}_{t}, \exists A^{0} \in \mathcal{F}_{t}^{0}, \mathbb{P}^{x}\left[A \triangle A^{0}\right]=0 .
$$

One of the most important examples of a eanonical strong Markov family arises as the family of laws of a RCLL version of a Feller process on a Hausdorff locallycompact topological space $E$ with a countable base (LCCB). We refer the reader to [35], Chapter $3, \S 2$ and $\S 3$, for details. The assumption of the Hausdorff property is made so that the state space $E$ is Polish - a property that will be needed for other aspects of the theory.

Many models used in finance (when viewed toghether with their factors) have the Feller property. Even more will form canonical strong Markov families, as defined above. A large class of examples are formed by the weak solutions of SDEs. 
More precisely, continuous solutions of uniquely-solvable local-martingale problems (in the sense of Stroock-Varadhan) with measurable coefficients define canonical strong Markov families (see Proposition 18.11, p. 344., in [29]).

Many processes with jumps will also fit our framework. For example, Lévy processes and various transformations thereof are Feller processes, as are solutions to a large class of SDEs driven by them (see Section 6., Chapter V, of [34] for details). In fact, it seems that all examples of Markov processes which do not give rise to an RCLL canonical strong Markov family, while abundant and important mathematically, feature serious pathologies as far as financial modelling is concerned.

Finally, even though this is not done here explicitly, there is nothing preventing "killed" processes from being included in the setup by the usual addition of a "cemetery" state to $E$.

In addition to the canonical strong Markov property, three mild further assumptions will be imposed on $\left(\mathbb{P}^{x}\right)_{x \in E}$. The first two correspond to the usual form most financial models take, while the third one is of technical nature and is satisfied in most models used in practice.

Finite Horizon. The first additional assumption simply encodes the standard trick which allows us to treat a finite-horizon, inhomogeneous Markov process in the infinite-horizon and homogeneous framework. We remind the reader that a set $E^{\prime} \subseteq E$ is said to be $\left(\mathbb{P}^{x}\right)_{x \in E^{-a b s o r b i n g}}$ if for each $x \in E^{\prime}, \mathbb{P}^{x}$ is the Dirac mass on the trajectory with constant value $x$.

Assumption 3.2 (Finite horizon).

1. $T_{t}=T_{0}-t$, for $t \leq T_{0}, \mathbb{P}^{x}$-a.s., for all $x \in E$, and

2. the set $E^{\prime}=\{0\} \times \mathbb{R}^{d} \times F \subseteq E$ is $\left(\mathbb{P}^{x}\right)_{x \in E \text {-absorbing. }}$

Absence of arbitrage. Unlike the $T$-component of $X$, which, thanks to Assumption 3.2 , can be interpreted as time-to-go, the $S$-components model risky-asset prices. The $F$-valued process $\eta$ plays the role of an external factor whose values drive the dynamics of $S$, but are not necessarily themselves tradeable in a financial market (stochastic volatility or macroeconomic indicators are two examples out of many). The fact that $S$ is assumed to be actively traded leads quite naturally to the economic assumption of absence of arbitrage, which comes in several variants in the literature, with the notion of No Free Lunch with Vanishing Risk (NFLVR) being probably the dominant one. We impose it here in an equivalent form by asking for the existence of an equivalent local-martingale measure. More precisely, for $x \in E$, let $\mathcal{M}^{x}$ denote the set of all probability measures $\mathbb{Q} \in \mathfrak{P}$, such that

1. $\mathbb{Q}$ and $\mathbb{P}^{x}$ are equivalent, and

2. the process $\left\{S_{t}\right\}_{t \in[0, \infty)}$ is a $\left(\mathbb{Q}, \mathbb{F}^{x}\right)$-local martingale.

Assumption 3.3 (NFLVR). For each $x \in E, \mathcal{M}^{x} \neq \emptyset$.

Thanks to [20, Theorem 5.3, p. 241], $\sigma$-marginales locally bounded from below are local martingales. Therefore, by [20, Theorem 1.1, p. 215], Assumption 3.3 is equivalent to the assumption of No Free Lunch with Vanishing Risk (NFVLR) in the financial model $\left\{S_{t}\right\}_{t \in[0, \infty)}$, on $\left(\Omega, \mathcal{F}, \mathbb{F}, \mathbb{P}^{x}\right)$, for all $x \in E$, whenever $S$ is locally 
bounded from below, $\mathbb{P}^{x}$-a.s., for all $x \in E$. As will be shown in the sequel, the local boundedness from below will be made a standing assumption.

Even though the main results of arbitrage theory were written under the usual conditions of right-continuity and completeness, we use the raw filtration $\mathbb{F}^{0}$ in the definition of $\mathcal{M}^{x}$. There is no loss of generality as far as the (true) martingale condition is concerned since, to switch to (the completion of) $\mathbb{F}$, it suffices to observe that $S$ is already $\mathbb{F}^{0}$-adapted and use Blumenthal's 0-1 law, i.e., (3.2). As for the localization, it is taken care of by our third additional assumption of local boundedness from below, introduced next.

Uniform local boundedness from below. For technical reasons which will soon become apparent, we need to impose another assumption on our canonical Markov family $\left(\mathbb{P}^{x}\right)_{x \in E}$. With $S$ being the "middle" component of the coordinate process $X$ on $D_{E}$, we set

Assumption 3.4 (Uniform local boundedness from below). For each $n \in \mathbb{N}$, there exists a constant $a_{n} \in \mathbb{R}$ such that

$$
\mathbb{P}^{x}\left[S^{\tau_{n}} \geq a_{n}\right]=1, \text { for all } x \in E \text {, where } \tau_{n}=\inf \left\{t \geq 0:\left|S_{t}\right| \geq n\right\},
$$

with the convention that all inequalities involving vector-valued processes are to be interpreted coordinatewise.

When applied to the process $S$ - interpreted as the asset-price process in a financial market - Assumption 3.4 of uniform local boundedness from below is very mild. Indeed, it covers most asset-price models used in practice, as they are mostly continuous or bounded from below (thanks to limited liability), and often both. Alternatively, a process with jumps bounded from below also satisfies Assumption 3.4.

The sequence $\left\{\tau_{n}\right\}_{n \in \mathbb{N}}$ of (3.3) is a prototypical example of a reducing sequence of stopping times for the local-martingale property, but such sequences are not enough in general. Indeed, it may happen that the "reason" for local martingality of a process is hidden in the full filtration, but, perhaps, not in its natural filtration (see, [41, p. 57] for an explicit example; for a glimpse of the general situation see the remainder of [41], as well as [28]). Under Assumption 3.4, however, the situation reverts back to its naive form:

Lemma 3.5. Let $\mathbb{Q} \in \mathfrak{P}$ be a probability measure equivalent to $\mathbb{P}^{x}$, for some $x \in E$. Under Assumption 3.4, $S$ is a $\left(\mathbb{Q}, \mathbb{F}^{0}\right)$-local martingale if and only if $S^{\tau_{n}}$ is a $\left(\mathbb{Q}, \mathbb{F}^{0}\right)$-martingale for each $n \in \mathbb{N}$.

Proof. The proof follows a well-known argument going back at least to [41]. Since it is short, we reformulate it here for convenience. As $\tau_{n}$ are stopping times and $\tau_{n}(\omega) \rightarrow \infty$, for all $\omega$, we only need to prove $S^{\tau_{n}}$ if a $\mathbb{Q}$-martingale as soon as $S$ it is a $\mathbb{Q}$-local martingale. Furthermore, we can assume, without loss of generality, that $S$ is real-valued. Thanks to the inequality $\left(S^{\tau_{n}}\right)^{*} \leq n+\left|S_{\tau_{n}}\right| \mathbf{1}_{\left\{\tau_{n}<\infty\right\}}$, it is enough to show that $S_{\tau_{n}} \mathbf{1}_{\left\{\tau_{n}<\infty\right\}} \in \mathbb{L}^{1}(\mathbb{Q})$, for each $n \in \mathbb{N}$. This follows, however, from the optional sampling theorem, since $S^{\tau_{n}}$ is a $\mathbb{Q}$-bounded-from-below $\mathbb{Q}$-local martingale, and, therefore, a $\mathbb{Q}$-bounded-from-below $\mathbb{Q}$-supermartingale. $\square$ 
3.2. Local-martingale measures form a controlled Markov family. Having described the model of the financial market, our next task is to show that the family $\mathcal{M}=\left(\mathcal{M}_{x}\right)_{x \in E}$ forms a controlled Markov family and that it is $G$-disintegrable for a large class of random variables $G$. Proofs of these claims, split into several auxiliary statements, will take the rest of this section.

Let us reiterate that Assumptions 3.2, 3.3 and 3.4 are in force throughout the section. Moreover, in the light of Assumption 3.2, we can (and do) assume without loss of generality that all random times in $\mathcal{T}$ are bounded. Indeed, for any initial state, the system will get absorbed in finite deterministic time.

Analyticity of the graph of $\mathcal{M}$. Our first task is to establish analyticity of the graph $\Gamma_{\mathcal{M}}=\left\{(x, \mathbb{Q}): \mathbb{Q} \in \mathcal{M}^{x}\right\}$. Here, as in the rest of this section, $\mathcal{Q}$ denotes the set of all probability measures $\mathbb{Q} \in \mathfrak{P}$ with $\mathbb{Q} \sim \mathbb{P}^{x}$ for some $x \in E$, and the sequence $\left\{\tau_{n}\right\}_{n \in \mathbb{N}}$ is as in Definition 3.3. For $x \in E$ and $n \in \mathbb{N}, \mathcal{M}_{n}^{x}$ is the set of all $\mathbb{Q} \sim \mathbb{P}^{x}$ under which (each coordinate of) $S^{\tau_{n}}$ is a $\mathbb{Q}$-martingale. Thanks to Lemma 3.5, we have the following equality

$$
\Gamma_{\mathcal{M}}=\cap_{n} \Gamma_{\mathcal{M}_{n}}, \text { where } \Gamma_{\mathcal{M}_{n}}=\left\{(x, \mathbb{Q}): \mathbb{Q} \in \mathcal{M}_{n}^{x}\right\} .
$$

This way, the question of analyticity of the graph $\Gamma_{\mathcal{M}}$ is reduced to the that of the sequence $\Gamma_{\mathcal{M}^{n}}$, defined via the (true) martingale property. It is in this step that the uniformity of local boundedness in Assumption 3.4 is important. Consequently, focusing on the (true) martingale property, we provide a simple characterization of the martingale property via a countable number of Borel operations; here $Q_{+}$denotes the set of all rational numbers in $[0, \infty)$.

Lemma 3.6. There exists a countable family $\left\{A_{q}^{n}\right\}_{q \in Q_{+}}^{n \in \mathbb{N}}$ such that $A_{q}^{n} \in \mathcal{F}_{q}^{0}$ for all $q \in Q_{+}, n \in \mathbb{N}$, with the following property: given $\mathbb{Q} \in \mathcal{Q}$, a bounded-from-below and $\mathbb{F}^{0}$-adapted $R C L L$ process $Y$ is an $\mathbb{F}$-martingale under $\mathbb{Q}$ if and only if

$$
\mathbb{E}^{\mathbb{Q}}\left[Y_{r} \mathbf{1}_{A_{q}^{n}}\right]=\mathbb{E}^{\mathbb{Q}}\left[Y_{q} \mathbf{1}_{A_{q}^{n}}\right], \text { for all } q \leq r \in Q_{+} \text {and } n \in \mathbb{N} .
$$

Proof. Necessity of (3.5) is clear, so we focus on sufficiency. The $\sigma$-algebra $\mathcal{F}_{q}^{0}$ is (bimeasurably) isomorphic to the Borel $\sigma$-algebra generated by the topology of the Polish space $D_{E}[0, q]$ of $E$-valued RCLL paths on $[0, q]$. Hence, it is countably generated, and we can choose an enumeration $\left\{A_{q}^{n}\right\}_{n \in \mathbb{N}}$ of a countable generating set. By adding all finite intersections if necessary, we can assume further, without loss of generality, that this generating set is a $\pi$-system, and that it contains $D_{E}$.

Since $Y_{0}$ is $\mathbb{Q}$-a.s.-constant for each $\mathbb{Q} \in \mathcal{Q}$, the condition (3.5) implies that, for $r \in Q_{+}$, we have $Y_{r} \in \mathbb{L}^{1}(\mathbb{Q})$ and

$$
\mathbb{E}^{\mathbb{Q}}\left[Y_{r} \mid \mathcal{F}_{q}^{0}\right]=Y_{q}, \mathbb{Q} \text {-a.s., for all } q \leq r \in Q_{+} .
$$

By Blumenthal's law (3.2) $\mathcal{F}_{q}^{0}$ and $\mathcal{F}_{q}$ differ in $\mathbb{Q}$-trivial sets only. Thus, the martingale property in (3.6) holds even if we replace $\mathcal{F}_{q}^{0}$ by $\mathcal{F}_{q}$. Also, for a fixed $r \in Q_{+}$, the family $\left\{Y_{q}\right\}_{q \in Q_{+}, q \leq r}$ is $\mathbb{Q}$-uniformly integrable. Hence, by the right continuity of $Y$, 
so is any family of the form $\left\{Y_{t}\right\}_{t \leq r}$, for $r \geq 0$. An approximation from the right yields that

$$
\mathbb{E}\left[Y_{t} \mid \mathcal{F}_{q}\right]=Y_{q}, \mathbb{Q} \text {-a.s., for each } t \geq 0, q \in Q_{+}, q \leq t .
$$

Another right approximation - this time in $q$ - and the backward martingale convergence theorem imply that

$$
\mathbb{E}\left[Y_{t} \mid \mathcal{F}_{s}\right]=Y_{s}, \mathbb{Q} \text {-a.s., for all } 0 \leq s \leq t<\infty .
$$

Therefore, $\left\{Y_{t}\right\}_{t \in[0, \infty)}$ is an $\mathbb{F}$-martingale and, a fortiori, an $\mathbb{F}^{0}$-martingale. $\mathbf{\square}$

After the local martingale property, we turn to the question of Borel-measurability of the measure-equivalence relation $\sim$ on $\mathfrak{P}$. The following auxiliary result appears to be well-known, but a precise reference has been hard to locate. We give a proof for completeness:

Lemma 3.7. Let $C$ be a fixed countable dense set in $D_{E}$ and let $\mathcal{B}$ be the (countable) family of all sets of the form $\cup_{i=1}^{m} B\left(x_{i}, 1 / n\right)$ for some finite family $x_{1}, \ldots, x_{m}$ in $C$ and some $n \in \mathbb{N}$. For $\mathbb{P}, \mathbb{Q} \in \mathfrak{P}$, we have $\mathbb{Q} \nless \mathbb{P}$ if and only if

$$
\exists N \in \mathbb{N} \quad \forall n \in \mathbb{N} \quad \exists B_{n} \in \mathcal{B} \quad \mathbb{P}\left[B_{n}\right] \leq \frac{1}{n} \text { and } \mathbb{Q}\left[B_{n}\right] \geq \frac{1}{N}
$$

Proof. If (3.7) holds it is standard to show that $\mathbb{Q} \nless \mathbb{P}$. Conversely, to show that $\mathbb{Q} \nless \mathbb{P}$ implies (3.7), we use the fact that all finite measures on Borel sets of a Polish space are regular $[1$, Theorem 12.7, p. 438$]$ to pick a compact set $K$ and a constant $N \in \mathbb{N}$, such that $\mathbb{P}[K]=0$ and $\mathbb{Q}[K] \geq 1 / N$. For $n \in \mathbb{N}$, let $\mathcal{B}_{n}$ denote the set of all open balls with centers in $C$ and radii $1 / n$, and let the family $\left\{\mathcal{B}_{n}^{K}\right\}_{n \in \mathbb{N}}$ of families of open balls be defined by

$$
\mathcal{B}_{n}^{K}=\left\{B \in \mathcal{B}_{n}: B \cap K \neq \emptyset\right\}, \text { for } n \in \mathbb{N} .
$$

By compactness, for each $n \in \mathbb{N}$ we can find a finite sub-collection $B_{1}^{n}, \ldots, B_{m_{n}}^{n}$ in $\mathcal{B}_{K}^{n}$ such that $K \subseteq B_{n}=\cup_{k=1}^{m_{n}} B_{k}^{n}$. Since $B_{n} \subseteq\{x \in X: d(x, K) \leq 1 / n\}$, we have $\mathbb{P}\left[B_{n}\right] \rightarrow 0$, as $n \rightarrow \infty$. On the other hand $\mathbb{Q}\left[B_{n}\right] \geq \mathbb{Q}[K] \geq 1 / N$, for all $n \in \mathbb{N}$.

The following statement can be derived as a direct consequence of $[21$, Theorem 58 , p. 52]. We include a short self-contained proof for completeness.

Corollary 3.8. The graph $\Gamma_{\sim}=\left\{(\mathbb{P}, \mathbb{Q}) \in \mathfrak{P}^{2}: \mathbb{P} \sim \mathbb{Q}\right\}$ of the measureequivalence relation $\sim$ is a Borel subset of $\mathfrak{P}^{2}$.

Proof. It suffices to notice that Lemma 3.7 states that we can express the graph $\Gamma_{\sim}=\{(\mathbb{P}, \mathbb{Q}): \mathbb{Q} \sim \mathbb{P}\}$ of the relation $\sim$ using only Borel operations starting from the sets of the form $\left\{(\mathbb{P}, \mathbb{Q}) \in \mathfrak{P}^{2}: \mathbb{P}[A] \leq \alpha\right\}$ and $\left\{(\mathbb{P}, \mathbb{Q}) \in \mathfrak{P}^{2}: \mathbb{Q}[A] \geq \beta\right\}$. That these subsets of $\mathfrak{P}^{2}$ are Borel measurable follows from a combination of the Portmanteau theorem and a monotone class argument.

The reader should compare our next proposition to a related, independent, discrete-time result (namely Lemma 4.8) in [16]. 
Proposition 3.9. The graph $\Gamma_{\mathcal{M}}$ is an analytic subset of $E \times \mathfrak{P}$.

Proof. By (3.4), we need to prove that $\Gamma_{\mathcal{M}_{n}}$ is analytic, for each $n \in \mathbb{N}$. We fix $n \in \mathbb{N}$ and observe that $\Gamma_{\mathcal{M}_{n}}=\Gamma_{1} \cap \Gamma_{2}$, where

$$
\Gamma_{1}=E \times\left\{\mathbb{Q} \in \mathfrak{P}: S^{\tau_{n}} \text { is a } \mathbb{Q} \text {-martingale }\right\} \text { and } \Gamma_{2}=\left\{(x, \mathbb{Q}) \in E \times \mathfrak{P}: \mathbb{Q} \sim \mathbb{P}^{x}\right\} \text {. }
$$

By Lemma 3.6, there exists a Borel set $\mathcal{R} \subseteq \mathfrak{P}$ such that

$$
\left\{\mathbb{Q} \in \mathfrak{P}: S^{\tau_{n}} \text { is a } \mathbb{Q} \text {-martingale }\right\} \cap \mathcal{Q}=\mathcal{R} \cap \mathcal{Q} .
$$

Since $\Gamma_{2} \subseteq E \times \mathcal{Q}$, it will be enough to show that $\Gamma_{2}$ is analytic.

It is a part of the definition of a canonical $\mathcal{T}$-Markov family that the map $x \mapsto$ $\mathbb{P}^{x} \in \mathfrak{P}$ is Borel. Therefore, so are its graph $\Gamma_{\mathbb{P}}=\left\{\left(x, \mathbb{P}^{x}\right): x \in E\right\} \subseteq E \times \mathfrak{P}$ and the product $\Lambda_{1}=\Gamma_{\mathbb{P}} \times \mathfrak{P}$. By Corollary 3.8, $\Lambda_{2}=E \times \Gamma_{\sim}$ is Borel, and, hence, so is

$$
\Lambda=\Lambda_{1} \cap \Lambda_{2}=\left\{\left(x, \mathbb{P}^{x}, \mathbb{Q}\right): \mathbb{Q} \sim \mathbb{P}^{x}\right\} .
$$

It remains to observe that $\Gamma_{2}$ is the (canonical) projection of $\Lambda$ onto the first and third coordinates and conclude that it is an analytic set in $E \times \mathfrak{P}$.

Closedness under concatenation and disintegrability. We remind the reader that $\mathcal{S}(\mathcal{M})$ denotes the set of all (universally measurable) kernels, and that each $\nu \in \mathcal{S}(\mathcal{M})$ can be interpreted as a universally-measurable map $x \mapsto \cup_{x \in E} \mathcal{M}^{x}$ with $\nu_{x} \in \mathcal{M}^{x}$ for all $x \in E$.

Proposition 3.10. For all $(x, \mathbb{Q}) \in \Gamma_{\mathcal{M}}, \tau \in \mathcal{T}$ and $\nu \in \mathcal{S}(\mathcal{P})$, we have

$$
\mathbb{Q} *_{\tau} \nu \in \mathcal{M}^{x} .
$$

Proof. Thanks to Assumption 3.4 and by stopping at some $\tau_{n}$ (as in (3.3)), we can assume that $S$ is bounded from below and a (true) martingale under each $\mathbb{Q} \in \cup_{x} \mathcal{M}^{x}$. Also, by considering each component separately, we may assume that $S$ is one-dimensional, i.e., that $d=1$.

We fix $(x, \mathbb{Q}), \tau$ and $\nu$ as in the statement, and note that, by direct computation, $\mathbb{Q} *_{\tau} \nu \sim \mathbb{P}^{x}$. Then, we pick a $0 \leq s \leq t$ and a bounded random variable $F \in \mathcal{F}_{\tau+s}$ and observe that, for all $\omega \in D_{E}$, the random variable $\omega^{\prime} \mapsto F\left(\omega *_{\tau} \omega^{\prime}\right)$ is $\mathcal{F}_{s}$-measurable. Therefore, by the $\mathbb{F}$-martingale property of $S$ under $\nu_{x}$, we have

$$
\int S_{t}\left(\omega^{\prime}\right) F\left(\omega *_{\tau} \omega^{\prime}\right) \nu_{x}\left(d \omega^{\prime}\right)=\int S_{s}\left(\omega^{\prime}\right) F\left(\omega *_{\tau} \omega^{\prime}\right) \nu_{x}\left(d \omega^{\prime}\right),
$$

for all $\omega \in D_{E}$ and $x \in E$. The identity $S_{r}\left(\omega^{\prime}\right)=S_{\tau+r}\left(\omega *_{\tau} \omega^{\prime}\right)$, valid for $\nu_{X_{\tau}(\omega)^{-}}$ almost all $\omega^{\prime}$ used with $r=s$ and $r=t$ implies that $\mathbb{E}^{\mathbb{Q} *_{\tau} \nu}\left[S_{\tau+t} F\right]=\mathbb{E}^{\mathbb{Q} *_{\tau} \nu}\left[S_{\tau+s} F\right]$. So,

$$
\mathbb{E}^{\mathbb{Q} *_{\tau} \nu}\left[S_{\tau+t} \mid \mathcal{F}_{\tau+s}\right]=S_{\tau+s}, \mathbb{Q} *_{\tau} \nu \text {-a.s., for all } 0 \leq s \leq t .
$$

In words, $S$ is a $\mathbb{Q} *_{\tau} \nu$-martingale "after $\tau$ ". On the other hand, $\mathbb{Q}$ and $\mathbb{Q} *_{\tau} \nu$ coincide on $\mathcal{F}_{\tau}$, which implies immediately that $S$ is a $\mathbb{Q} *_{\tau} \nu$-martingale "before $\tau$ ". Thanks 
to the optional sampling theorem, the two properties combine, and we conclude that $S$ is a $\mathbb{Q} *_{\tau} \nu$-martingale. Indeed, for a bounded stopping time $\kappa$ we have the following chain of inequalities, where all the expectations are under $\mathbb{Q} *_{\tau} \nu$ :

$$
\begin{aligned}
\mathbb{E}\left[S_{\kappa}\right] & =\mathbb{E}\left[S_{\kappa \wedge \tau} \mathbf{1}_{\{\kappa \leq \tau\}}+S_{\kappa \vee \tau} \mathbf{1}_{\{\kappa>\tau\}}\right]=\mathbb{E}\left[\mathbb{E}\left[S_{\tau} \mid \mathcal{F}_{\kappa \wedge \tau}\right] \mathbf{1}_{\{\kappa \leq \tau\}}+\mathbb{E}\left[S_{\kappa \vee \tau} \mathbf{1}_{\{\kappa>\tau\}} \mid \mathcal{F}_{\tau}\right]\right] \\
& =\mathbb{E}\left[S_{\tau} \mathbf{1}_{\{\kappa \leq \tau\}}\right]+\mathbb{E}\left[S_{\tau} \mathbf{1}_{\{\kappa>\tau\}}\right]=\mathbb{E}\left[S_{\tau}\right] .
\end{aligned}
$$

Proposition 3.11. The family $\left(\mathcal{M}^{x}\right)_{x \in E}$ satisfies the condition (2.11) of Proposition 2.5.

Proof. We pick $(x, \mathbb{Q}) \in \Gamma_{\mathcal{M}}, \tau \in \mathcal{T}$, and recall that $\tau$ is bounded. As in the proof of Proposition 3.10, we assume that $S$ is a one-dimensional $\mathbb{Q}$-martingale bounded from below, for each $\mathbb{Q} \in \cup_{x \in E} \mathcal{M}^{x}$.

Let $q_{x}$ be the $\mathbb{Q}$-regular conditional distribution of $\theta_{\tau}$, given $X_{\tau}=x$. By constructing the Radon-Nikodym density, for example, we immediately note that $q_{x} \sim \mathbb{P}^{x}$, for $\mathbb{Q} \circ X_{\tau}^{-1}$-almost all $x \in E$. Given a stopping time $\sigma \in \mathcal{T}$, we set $\kappa=\tau+\sigma \circ \theta_{\tau}$. Since both $\tau$ and $\sigma$ are stopping times for the shifted raw filtration $\left\{\mathcal{F}_{t+\varepsilon}^{0}\right\}_{t \in[0, \infty)}$, for each $\varepsilon>0$, Galmarino's test (see, e.g., Exercise 4.21, p. 47 in [35]), implies that $\kappa$ has the same property, and so, $\kappa \in \mathcal{T}$. Therefore, $\mathbb{E}^{\mathbb{Q}}\left[S_{\kappa} \mid \sigma\left(X_{\tau}\right)\right]=S_{\tau}$, $\mathbb{Q}$-a.s. Moreover, $S_{\kappa}=S_{\sigma} \circ \theta_{\tau}$, and so,

$$
\mathbb{E}^{\mathbb{Q}}\left[f\left(X_{\tau}\right) S_{\tau}\right]=\mathbb{E}^{\mathbb{Q}}\left[f\left(X_{\tau}\right) S_{\kappa}\right]=\mathbb{E}^{\mathbb{Q}}\left[f\left(X_{\tau}\right) \int S_{\sigma}\left(\omega^{\prime}\right) q_{X_{\tau}}\left(d \omega^{\prime}\right)\right],
$$

for all bounded Borel functions $f: E \rightarrow \mathbb{R}$. In particular, it follows that

$$
\int S_{0}\left(\omega^{\prime}\right) q_{X_{\tau}(\omega)}\left(d \omega^{\prime}\right)=S_{\tau}(\omega)=\int S_{\sigma}\left(\omega^{\prime}\right) q_{X_{\tau}(\omega)}\left(d \omega^{\prime}\right), \text { for } \mathbb{Q} \text {-almost all } \omega \in D_{E} .
$$

Therefore $S_{\sigma}$ and $S_{0}$ have the same expectation under $q_{x}$, for $\mathbb{Q} \circ X_{\tau}^{-1}$-almost all $x$, with the exceptional set possibly depending on the stopping time $\sigma$. By Lemma 3.6, however, it suffices to consider a countable collection of stopping times of the form $\sigma=q \mathbf{1}_{A_{q}^{n}}+r \mathbf{1}_{\left(A_{q}^{n}\right)^{c}}$, for rational $q<r$ and $A_{q}^{n}$ as in the statement of Lemma 3.6 we conclude that $S$ is $q_{x}$-martingale for $\mathbb{Q} \circ X_{\tau}^{-1}$-almost all $x \in E$. $\mathrm{Q}$

3.3. Lower hedging. We adopt the framework and notation of subsection 3.1 and turn to the problem of lower hedging (sub-hedging) in a financial market. First, we define a random variable $G$ - with a pointwise shift-invariance property - which will play a role of the contingent claim to be sub-replicated, The main ingredient is the following simple observation which states, loosely, that our state space admits a "limit superior" (a weaker, but measurable, notion similar to that of a Banach limit).

Lemma 3.12. There exists a measurable functional $L: D_{E} \rightarrow E$ such that

1. $L(\omega)=\lim _{t \rightarrow \infty} X_{t}(\omega)$, whenever the limit exists, and

2. $L\left(\theta_{t}(\omega)\right)=L(\omega)$, for all $\omega \in D_{E}$ and all $t \geq 0$.

Proof. $E$ is a metrizable noncompact LCCB space, so its Alexandroff (one-point) compactification is Polish (see [1, Theorem 3.44, p. 92]). Therefore, it can be embedded into the Hilbert cube $H=[0,1]^{\mathbb{N}}$ as a compact set. In fact, we identify $E$ with its copy in $H$, and we denote the image of the "infinity point" by $\infty$. Let $p_{n}$, $n \in \mathbb{N}$, denote the coordinate projections on $H$. For $\omega \in D_{E}$ we define $L^{\prime}(\omega) \in H$ by 
requiring that

$$
p_{n}\left(L^{\prime}(\omega)\right)=\limsup _{t \rightarrow \infty} p_{n}\left(X_{t}(\omega)\right) \text {, for all } n \in \mathbb{N},
$$

so that, immediately, we have $L^{\prime}\left(\theta_{t}(\omega)\right)=L(\omega)$, for all $t \geq 0$ and $\omega \in D_{E}$. The continuity of each $p_{n}$ and the RCLL property of $X_{t}$ guarantee the Borel measurability of the map $\omega \mapsto \lim \sup _{t \rightarrow \infty} p_{n}\left(X_{t}(\omega)\right)$, for each $n \in \mathbb{N}$, and, therefore, of the map $L^{\prime}$. Thanks to the compactness of $E \cup\{\infty\}$, a RCLL path $t \mapsto X_{t}$ in $E$ will converge to $x \in E \cup\{\infty\}$ if and only if each of the $[0,1]$-valued paths $p_{n}\left(X_{t}\right)$ converges to $p_{n}(x)$ as $t \rightarrow \infty$. It follows that $L^{\prime}(\omega)=\lim _{t \rightarrow \infty} X_{t}(\omega)$, whenever this limit exists and that $L^{\prime}(\omega) \in E \cup\{\infty\}$ otherwise. It remains to remap $\infty$ to an arbitrary point in $E$, i.e., to define the map $L$ by $L=\kappa \circ L^{\prime}$, where $\kappa$ is a measurable "retraction" $\kappa: E \cup\{\infty\} \rightarrow E$.

With $L$ as in Lemma 3.12 above and a Borel function $\varphi: E \rightarrow \mathbb{R}$, we set

$$
G(\omega)=\tilde{\varphi}(L(\omega)),
$$

where $\tilde{\varphi}(t, S, \eta)=\varphi(S, \eta)$, for all $(t, S, \eta) \in E$. Since under each $\mathbb{P}^{x}$, the coordinate process $X=(T, S, \eta)$ gets absorbed after $T_{0}$ units of time, and the absorption point is $L(\omega), \mathbb{P}^{x}$-a.s., it is clear that $G$ can be interpreted as the value of a derivative claim written on the risky asset $S$ as well as the factor $\eta$, with maturity $T_{0}$. With the standing interpretation of the first coordinate process $T$ as "time to go", $G$ should be understood $\mathbb{P}^{x}$-a.s. as a composition of a Borel function (the payoff function) with the values of the risky asset $S$ and the factor $\eta$ at maturity $T_{0}$ units of time from now. Moreover, we have $G \circ \theta_{t}=G$, for all $t$; indeed, this property is directly inherited from $L$.

Under the minimal assumption that $\mathbb{E} \mathbb{Q}^{[}\left[G^{-}\right]<\infty$, for all $\mathbb{Q} \in \cup_{x} \mathcal{M}^{x}$, for a given $x=(T, S, \eta) \in[0, \infty) \times \mathbb{R}^{d} \times F$, we define the lower-hedging price of $G$ as

$$
v(T, S, \eta)=\inf _{\mathbb{Q} \in \mathcal{M}^{T, S, \eta}} \mathbb{E}^{\mathbb{Q}}[G]
$$

One usually (and more naturally) defines the lower hedging price $v(x)=v(t, S, \eta)$ as follows:

$$
v(T, S, \eta)=\sup \left\{y \in \mathbb{R}: \exists H \in \mathcal{A}^{T, S, \eta}, y+\int_{0}^{T} H_{u} d S_{u} \geq \varphi\left(S_{T}, \eta_{T}\right), \mathbb{P}^{T, S, \eta}-\text { a.s }\right\},
$$

where $\mathcal{A}^{T, S, \eta}$ denotes the set of all admissible strategies. The two formulations are equivalent (see [20, Theorem 5.12, p. 246]), but we prefer the one in (3.8) as it fits our framework much better.

ThEOREM 3.13 (DPP for lower hedging). Under Assumptions 3.1, 3.2, 3.3 and 3.4, and with $v$ defined in (3.8), we have

1. The value function $v:[0, \infty) \times \mathbb{R}^{d} \times F \rightarrow[-\infty, \infty]$ is universally measurable and the dynamic-programming equation (DPP) holds:

$$
v(T, S, \eta)=\inf _{\mathbb{Q} \in \mathcal{M}^{T, S, \eta}} \mathbb{E}^{\mathbb{Q}}\left[v\left(T-\tau, S_{\tau}, \eta_{\tau}\right)\right], \quad v(0, S, \eta)=\varphi(\eta, S),
$$

for all $\mathbb{F}$-stopping times $\tau \leq T$, where we define $\mathbb{E}^{\mathbb{Q}}[Y]=\infty$, as soon as $\mathbb{E}^{\mathbb{Q}}\left[Y^{+}\right]=\infty$. 
2. For $\varepsilon>0$, an $\varepsilon$-optimal $\hat{\mathbb{Q}}^{T, S, \eta} \in \mathcal{M}^{T, S, \eta}$ can be associated to each $(T, S, \eta) \in$ $[0, \infty) \times \mathbb{R}^{d} \times E$ in a universally-measurable way.

Proof. According to Propositions 3.9 and 3.10, the pair $(\mathcal{M}, \mathcal{T})$ forms a controlled Markov family, with $\mathcal{T}$ denoting the set of all bounded $\mathbb{F}$-stopping times. Moreover, since $G \circ \theta_{t}=G$, the statement of Proposition 3.11 guarantees that the conditions of Proposition 2.5 are satisfied; hence, $G$ is $(\mathcal{T}, \mathcal{M})$-disintegrable. That, in turn, allows for our main abstract result, Theorem 2.4 , to be applied.

3.4. Utility maximization. The utility-maximization problem - its generalized dual formulation, to be more precise - can be phrased in the framework almost identical to that of subsection (3.3). Indeed, given $z \geq 0$ we pose the generalized dual utility-maximization problem:

$$
v(T, S, \eta, z)=\inf _{\mathbb{Q} \in \mathcal{M}^{T, S, \eta}} \mathbb{E}^{T, S, \eta}\left[V\left(S_{T}, \eta_{T}, z \frac{d \mathbb{Q}}{d \mathbb{P}^{T, \eta, S}}\right)\right],
$$

where $\mathbb{E}^{T, S, \eta}$ denotes the expectation under $\mathbb{P}^{T, S, \eta}$, and $V:[0, \infty)^{d} \times F \times[0, \infty) \rightarrow \mathbb{R}$ is a Borel function with the property that $\mathbb{E}^{T, S, \eta}\left[V^{-}\left(S_{T}, \eta_{T}, z \frac{d \mathbb{Q}}{d \mathbb{P}^{T, \eta, S}}\right)\right]<\infty$, for all $\mathbb{Q} \in \mathcal{M}^{T, S, \eta}$.

Unfortunately, the objective function in 3.9 is not of the form $\mathbb{E}^{\mathbb{Q}}[G]$ for a random variable $G$ defined on $D_{E}$, so our main abstract result cannot be directly applied. It will be possible to do that, however, if we include $[0, \infty)$ as an additional factor in the definition of the state space:

$$
\tilde{E}=E \times[0, \infty)=[0, \infty) \times[0, \infty)^{d} \times F \times[0, \infty) .
$$

We identify the space $D_{\tilde{E}}$ with the product space $D_{E} \times D_{[0, \infty)}$, where $E=[0, \infty) \times$ $[0, \infty)^{d} \times F$ is the state space of the lower-hedging problem. To simplify the notation, the coordinate process on $E$ will be denoted by $X$ and that on $\tilde{E}$ by $\tilde{X}=(X, Z)$; a generic point on $E$ is $x=(T, S, \eta)$, while $\tilde{x}=(x, z)$ is a generic point in $\tilde{E}$. For a fixed element $x \in E$ and a martingale measure $\mathbb{Q} \in \mathcal{M}^{x}$, using $D_{E}$ as the underlying probability space, we construct a RCLL version of the Radon-Nikodym density $Z^{\mathbb{Q}}$ of $\mathbb{Q}$ with respect to $\mathbb{P}^{x}$ :

$$
Z_{t}^{\mathbb{Q}}=\mathbb{E}^{x}\left[\frac{d \mathbb{Q}}{d \mathbb{P}^{x}} \mid \mathcal{F}_{t}\right], \text { for } t \geq 0 .
$$

For $x \geq 0$, the $\mathbb{P}^{x}$-law of the of vector of processes $\left(X, z Z^{\mathbb{Q}}\right)=\left(T, S, \eta, z Z^{\mathbb{Q}}\right)$ on $D_{E}$ defines a Borel probability measure on $D_{\tilde{E}}$, which we denote by $\mathbb{P}^{T, S, \eta, z ; Q}$, or, simply, by $\mathbb{P}^{x, z ; \mathbb{Q}}$. It is characterized by the following equality

$$
\mathbb{E}^{\mathbb{P}^{x, z ; \mathbb{Q}}}[G(X ., Z .)]=\mathbb{E}^{x}\left[G\left(X ., z Z_{.}^{\mathbb{Q}}\right)\right],
$$

valid for all bounded Borel $G: D_{\tilde{E}} \rightarrow \mathbb{R}$. For $(x, z) \in E^{\prime}$, we define

$$
\tilde{\mathcal{P}}^{x, z}=\left\{\mathbb{P}^{x, z ; \mathbb{Q}}: \mathbb{Q} \in \mathcal{M}^{x}\right\} \subseteq \tilde{\mathfrak{P}}
$$

where $\tilde{\mathfrak{P}}$ denotes the set of all probability measures on $D_{\tilde{E}}$. The natural filtration generated by $\tilde{X}$ on $D_{\tilde{E}}$ is denoted by $\tilde{\mathbb{F}}^{0}$, and its right-continuous hull by $\tilde{\mathbb{F}}$; their raw 
and right-continuous sub-filtrations, generated by $X$, will be denoted by $\tilde{\mathbb{F}}^{X ; 0}$ and $\tilde{\mathbb{F}}^{X}$, respectively. As usual, $Q_{+}$is the set of nonnegative rational numbers.

Proposition 3.14. The family $\tilde{\mathcal{P}}^{x, z},(x, z) \in \tilde{E}$ is closed under concatenation.

Proof. We pick a probability $\mathbb{P}^{x_{0}, z_{0} ; \mathbb{Q}_{0}}$, a stopping time $\tilde{\tau}$ and a kernel $\left(\hat{\mathbb{P}}^{x, z: \hat{\mathbb{Q}}^{x, z}}\right)_{x, z}$ into elements of $\tilde{\mathcal{P}}$. It is immediate that that $\left(\hat{\mathbb{Q}}^{x, z}\right)_{x, z}$ is also a kernel, from $\tilde{E}$ into probability measures on $D_{\tilde{E}}$. To avoid multiple levels of indexation, we shorten $\mathbb{P}^{x_{0}, z_{0} ; \mathbb{Q}_{0}}$ to $\mathbb{P}^{0}$ and denote by $\mathbb{E}^{0}$ the corresponding expectation operator. Also, the process $Z^{\mathbb{Q}^{0}}$ will be denoted simply by $Z^{0}$.

For $q \in Q_{+}$, we define $\tilde{B}_{q}=\{\tilde{\tau}<q\} \in \tilde{\mathcal{F}}_{q}^{0}$. Since $Z^{0}$ is $\tilde{\mathbb{F}}^{X}$-adapted, there exists an $\tilde{\mathcal{F}}_{q}^{X}$-measurable set $\tilde{B}_{q}^{\prime}$ such that $\mathbb{P}^{0}\left[\tilde{B}_{q}^{\prime} \triangle \tilde{B}_{q}\right]=0$. Each set $\tilde{B}_{q}^{\prime}, q \in Q_{+}$can be further identified with an event $B_{q} \in \mathcal{F}_{q}$ on $D_{E}$ such that, with the $\mathbb{F}$-stopping time $\tau$ on $D_{E}$ defined by

$$
\tau(\omega)=\sup \left\{q \in Q_{+}: \omega \in B_{q}\right\},
$$

we have, for each bounded Borel $H: D_{\tilde{E}} \rightarrow \mathbb{R}$,

$$
\mathbb{E}^{0}\left[H\left(X_{\tilde{\tau} \wedge}, Z_{\tilde{\tau} \wedge}\right)\right]=\mathbb{E}^{x}\left[H\left(X_{\tau \wedge \cdot}, z Z_{\tau \wedge \cdot}^{\mathbb{Q}}\right)\right],
$$

where the expectation on the left is over $D_{\tilde{E}}$ and the one on the right over $D_{E}$.

We would like to prove that $\mathbb{P}^{0} *_{\tilde{\tau}} \hat{\mathbb{P}}^{\cdot}=\mathbb{P}^{x_{0}, z_{0} ; \hat{\mathbb{Q}}}$, for some $\hat{\mathbb{Q}} \in \mathcal{M}^{x_{0}}$, with $\left.\mathbb{Q}\right|_{\mathcal{F}_{\tau}}=$ $\mathbb{Q}_{0} \mid \mathcal{F}_{\tau}$. Consider the family $\mathcal{A}$ of all random variables of the product form $L \times\left(H \circ \theta_{\tilde{\tau}}\right)$ where $L=L(X ., Z$. $), H=H\left(X ., Z\right.$.) are bounded and Borel on $D_{\tilde{E}}$, and $L$ is $\mathcal{F}_{\tilde{\tau}^{-}}^{0}$ measurable. By the monotone-class theorem, it will be enough to show that the two measures act on $\mathcal{A}$ in the same way. For $L H \circ \theta_{\tilde{\tau}} \in \mathcal{A}$ we have

$$
\mathbb{E}^{\mathbb{P}^{0} *_{\tilde{\tau}} \hat{\mathbb{P}}^{*}}\left[L \times\left(H \circ \theta_{\tilde{\tau}}\right)\right]=\mathbb{E}^{0}\left[\operatorname{Lh}\left(X_{\tilde{\tau}}, z_{0} Z_{\tilde{\tau}}^{0}\right)\right],
$$

where $h(x, z)=\mathbb{E}^{\hat{\mathbb{P}}^{x, z}}[H]=\mathbb{E}^{x}\left[H\left(X ., z Z^{\mathbb{Q}^{x, z}}\right)\right]$, i.e.,

$$
\mathbb{E}^{\mathbb{P}^{0} *_{\tilde{\tau}} \hat{\mathbb{P}}^{\cdot}}\left[L \times\left(H \circ \theta_{\tilde{\tau}}\right)\right]=\int L(\omega) \mathbb{E}^{X \tilde{\tau}(\omega)}\left[H\left(X .\left(\omega^{\prime}\right), z_{0} Z_{\tau}^{0}(\omega) Z^{\mathbb{Q}^{X}(\omega), Z_{\tau}^{0}(\omega)}\left(\omega^{\prime}\right)\right)\right] \mathbb{P}[d \omega] .
$$

If, for $t \geq 0$, we set $\hat{Z}_{t}=Z_{t \wedge \tau}^{0}, Z_{t-t \wedge \tau}^{\mathbb{Q}^{X_{\tau}, Z_{\tau}^{0}}}$, it is clear that $\hat{Z}$ is an $\mathbb{F}$-adapted, RCLL martingale. Moreover, it has the easy-to-verify property that (each component of) $\hat{Z} S^{\tau_{n}}$ is a martingale on $D_{E}$ for each $n \in \mathbb{N}$, with $\left\{\tau_{n}\right\}_{n \in \mathbb{N}}$ given in (3.3). Finally, since $\hat{Z}_{\infty}>0, \mathbb{P}^{x}$-a.s., there exists a probability measure $\widehat{\mathbb{Q}} \in \mathcal{M}^{x}$ such that $\hat{Z}=Z^{\widehat{\mathbb{Q}}}$. The reader will now readily check that $\mathbb{E}^{\mathbb{P}^{0} * \tilde{\tau} \hat{\mathbb{P}}^{*}}\left[L \times\left(H \circ \theta_{\tilde{\tau}}\right)\right]=\mathbb{E}^{x_{0}, z_{0}}: \hat{\mathbb{Q}}\left[L \times\left(H \circ \theta_{\tilde{\tau}}\right)\right]$,

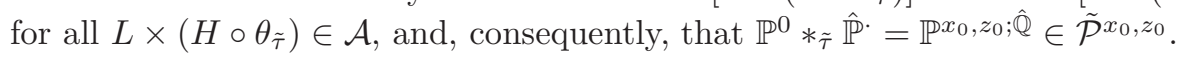

Using the corresponding statement for the family $\mathcal{M}^{x}$, namely Proposition 3.11 and the ideas from the above proof of Proposition 3.14, we get the following result:

Proposition 3.15. The family $\tilde{\mathcal{P}}^{x, z},(x, z) \in E^{\prime}$ is $G$-disintegrable, for all $G$ satisfying (2.10).

Finally, we turn to the proof of analyticity of the graph $\Gamma_{\tilde{\mathcal{P}}}$ of $\left(\mathcal{P}^{x, z}\right)_{x, z}$. The reader will note that some parts of the construction used in the proof of Proposition 
3.16 below are quite similar to the central argument in the proof of existence of a jointly measurable Radon-Nikodym derivative in Theorem 58, p. 52 of [21].

Proposition 3.16. The graph $\Gamma_{\tilde{\mathcal{P}}}=\left\{(x, z, \mathbb{P}):(x, z) \in \tilde{E}, \mathbb{P} \in \tilde{\mathcal{P}}^{x, z}\right\} \subseteq \tilde{E} \times \tilde{\mathfrak{P}}$ is analytic.

Proof. Let $\hat{\mathfrak{P}}$ denote the set of all pairs $(\mathbb{P}, \mathbb{Q})$ of probability measures on $D_{E}$ with

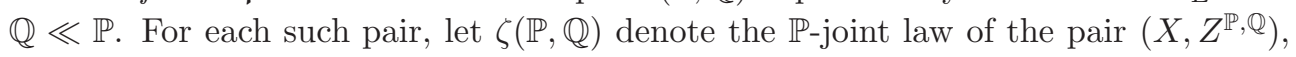
on $D_{\tilde{E}}$, where $Z^{\mathbb{P}, \mathbb{Q}}$ is the RCLL version of the martingale

$$
Z_{t}^{\mathbb{P}, \mathbb{Q}}=\mathbb{E}^{\mathbb{P}}\left[\frac{d \mathbb{Q}}{d \mathbb{P}} \mid \mathcal{F}_{t}\right] .
$$

Using the stability under Borel maps of the analytic sets, it is enough to show that the map $\zeta$ is Borel. As already commented on in subsection 2.4, part (2), by the classical result [43, Lemma 2.3, p. 194] of Varadarajan, it suffices to show that $\zeta$ is customarily Borel measurable, i.e., that the map

$$
(\mathbb{P}, \mathbb{Q}) \mapsto \zeta(\mathbb{P}, \mathbb{Q})[B],
$$

is Borel, for each Borel $B$ on $D_{\tilde{E}}$. The $\pi-\lambda$ theorem can be used to reduce this further to product cylinders. More precisely, it is enough to show that the map

$$
(\mathbb{P}, \mathbb{Q}) \mapsto \mathbb{P}\left[\left(X_{t_{1}}, Z_{t_{1}}^{\mathbb{P}, \mathbb{Q}}\right) \in C_{1} \times D_{1}, \ldots,\left(X_{t_{n}}, Z_{t_{n}}^{\mathbb{P}, \mathbb{Q}}\right) \in C_{n} \times D_{n}\right]
$$

is Borel, for each $n \in \mathbb{N}$, and all $C_{1}, \ldots, C_{n} \in \mathcal{B}(E)$ and $D_{1}, \ldots, D_{n} \in \mathcal{B}(\mathbb{R})$. It turns out to be more convenient, but equally valid, to consider bounded continuous functions $f: \tilde{E}^{n} \rightarrow \mathbb{R}$ and the maps

$$
(\mathbb{P}, \mathbb{Q}) \mapsto \mathbb{E}^{\mathbb{P}}\left[f\left(\left(X_{t_{1}}, Z_{t_{1}}^{\mathbb{P}, \mathbb{Q}}\right), \ldots\left(X_{t_{n}}, Z_{t_{n}}^{\mathbb{P}, \mathbb{Q}}\right)\right)\right] .
$$

By approximation, it suffices to show that maps of the form

$$
(\mathbb{P}, \mathbb{Q}) \mapsto \mathbb{E}^{\mathbb{P}}\left[F\left(X_{t_{1}}, X_{t_{2}}, \ldots, X_{t_{n}}\right) \rho\left(Z_{t_{1}}^{\mathbb{P}, \mathbb{Q}}, \ldots, Z_{t_{n}}^{\mathbb{P}, \mathbb{Q}}\right)\right]
$$

are Borel, for all nonnegative and bounded $F$ and continuous and convex $\rho$. We can write $\rho$ as a supremum of a countable number of affine functions on $\mathbb{R}^{n}$, so the problem reduces to the Borel-measurability of the map of the form

$$
(\mathbb{P}, \mathbb{Q}) \mapsto \mathbb{E}^{\mathbb{P}}\left[F\left(X_{t_{1}}, X_{t_{2}}, \ldots, X_{t_{n}}\right) Z_{t}^{\mathbb{P}, \mathbb{Q}}\right],
$$

for all $t \geq 0$. Using the fact that $\mathcal{F}_{t}^{0}=D_{E}[0, t]$, we can construct a nested sequence $\left\{\mathcal{K}_{n}\right\}_{n \in \mathbb{N}}$ of finite partitions of $\mathcal{F}_{t}^{0}$ with the property that $\sigma\left(\cup_{n} \mathcal{K}_{n}\right)=\mathcal{F}_{t}^{0}$. For each $n \in \mathbb{N}$ and each pair $(\mathbb{P}, \mathbb{Q}) \in \mathfrak{P}^{2}$, we define a $\sigma\left(\mathcal{K}_{n}\right)$-measurable random variable $Z_{n}^{\mathbb{P}, \mathbb{Q}}$ on $D_{E}$ as follows:

$$
Z_{n}^{\mathbb{P}, \mathbb{Q}}=\sum_{i=1}^{\left|\mathcal{K}_{n}\right|} \frac{\mathbb{Q}\left[A_{i}^{n}\right]}{\mathbb{P}\left[A_{i}^{n}\right]} \mathbf{1}_{A_{i}^{n}},
$$

with $\mathcal{K}_{n}=\left\{A_{1}^{n}, \ldots, A_{\left|\mathcal{K}_{n}\right|}^{n}\right\}$ and $\frac{\mathbb{Q}\left[A_{i}^{n}\right]}{\mathbb{P}\left[A_{i}^{n}\right]}=0$, as soon as $\mathbb{P}\left[A_{i}^{n}\right]=0$. The martingaleconvergence theorem implies that $Z_{n}^{\mathbb{P}, \mathbb{Q}} \rightarrow Z_{t}^{\mathbb{P}, \mathbb{Q}}, \mathbb{P}$-a.s., which, in turn, establishes the Borel-measurability in (3.14) and completes the proof. 
Propositions 3.14, 3.15 and 3.16 allow us to apply the abstract DPP of Theorem 2.4 in the present setting.

Theorem 3.17 (DPP for utility maximization). Under Assumptions 3.1, 3.2, 3.3 and 3.4, and with $v$ and $V$ as in equation (3.9) and below it, we have

1. The value function $v:[0, \infty) \times \mathbb{R}^{d} \times F \times[0, \infty) \rightarrow[-\infty, \infty]$ is universally measurable and the dynamic-programming equation (DPP) holds:

$$
v(T, S, \eta, z)=\inf _{\mathbb{Q} \in \mathcal{M}^{T, S, \eta}} \mathbb{E}^{\mathbb{P}}\left[v\left(T-\tau, S_{\tau}, \eta_{\tau}, z Z_{\tau}^{\mathbb{Q}}\right)\right], \quad v(0, S, \eta, z)=V(\eta, S, z),
$$

for all $\mathbb{F}$-stopping times $\tau \leq T$, where $Z_{\tau}^{\mathbb{Q}}=\mathbb{E}^{\mathbb{P}^{T, S, \eta}}\left[\frac{d \mathbb{Q}}{d \mathbb{P}^{T, S, \eta}} \mid \mathcal{F}_{\tau}\right]$ and with the convention that $\mathbb{E}^{\mathbb{Q}}[Y]=\infty$, as soon as $\mathbb{E}^{\mathbb{Q}}\left[Y^{+}\right]=\infty$.

2. For $\varepsilon>0$, an $\varepsilon$-optimal $\hat{\mathbb{Q}}^{T, S, \eta, z} \in \mathcal{M}^{T, S, \eta}$ can be associated to each $(T, S, \eta, z)$ $\in[0, \infty) \times \mathbb{R}^{d} \times E \times[0, \infty)$ in a universally-measurable way.

We focus on the dual formulation of the utility-maximization problem in this paper not only because it fits our framework, but also because it is often much easier to work with in practice. For the reader interested in the primal problem, here are a few words about its relationship with the dual problem and the DPP.

The classical utility-maximization theorem with random endowment in as described in [19] and adadpted to fit our notation comes with the function $V$ of the form

$$
V(S, \eta, z)=\tilde{U}(z)+z \varphi(S, \eta)
$$

where $\varphi$ is a bounded Borel function and $\tilde{U}(z)=\sup _{\xi \geq 0}(U(\xi)-\xi z)$ is the dual of a utility function $U$, i.e., an increasing and strictly concave $C^{1}$-map $U:(0, \infty) \rightarrow \mathbb{R}$, with $U^{\prime}(0+)=\infty$ and $U^{\prime}(\infty)=0$. Additionally, the condition of reasonable asymptotic elasticity $\mathrm{AE}[U]<1$ is imposed, where $\mathrm{AE}[U]=\limsup _{x \rightarrow \infty} x U^{\prime}(x) / U(x)<1$ when $U(x)>0$ for large enough $x$ and $\operatorname{AE}[U]=0$, otherwise. Under these conditions, and a suitable finiteness assumption, the authors of [19] show that the following conjugate relationship holds

$$
u(T, S, \eta, \xi)=\inf _{z \in Q_{+}}(v(T, S, \eta, z)-\xi z),
$$

where $u$ is the value function of the primal utility-maximization problem given by

$$
u(T, S, \eta, \xi)=\sup _{H \in \mathcal{A}^{T, S, \eta}} U\left(\xi+\int_{0}^{T} H_{u} d S_{t}+\varphi\left(S_{T}, \eta_{T}\right)\right),
$$

with $\mathcal{A}^{T, S, \eta}$ denoting the set of admissible portfolio processes, and where $T, S, \eta$ and $\xi$ range over the problem's effective domain. The infimum in (3.15) is taken over a countable set, so the conclusion of universal measurability transfers directly from $v$ to $u$. The same relationship can be used to show that the function $u$ satisfies the appropriate version of the DPP.

Appendix A. Some notions from descriptive set theory. For technical reasons, some basic concepts from descriptive set theory are needed. The following few 
paragraphs provide a (necessarily inadequate) review of the most prominent notions and facts; we refer the reader to Chapter 7 of [6], or the textbooks [39] and [30], for a thorough treatment.

A Polish space is a separable topological space whose topology can be induced by a complete metric. The main examples include all countable discrete spaces, as well as Euclidean spaces $\mathbb{R}^{d}, d \in \mathbb{N}$, in addition to a host of "infinite-dimensional" spaces such as the Hilbert space $[0,1]^{\mathbb{N}}$, the Baire space $\mathbb{N}^{\mathbb{N}}$, or any separable Banach space, all with the usual topologies. Compact metric spaces as well as locally-compact Hausdorff spaces with a countable base (LCCB) are Polish; so are countable products (with the product topology) of Polish spaces and all their $G_{\delta}$ (and, in particular, open or closed) subsets.

A subset $A$ of a Polish space $X$ is called analytic if it can be realized as a projection of a Borel subset of $X \times \mathbb{R}$ onto $X$. Borel subsets of Polish spaces are analytic, but, unless the space is countable, one can always find non-Borel analytic sets. The family of all analytic sets is closed under countable unions and intersections, as well as direct and inverse images of Borel maps. It is, generally, not closed under complementation. In fact, a complement of an analytic set, known as a co-analytic set, is itself analytic if and only if it is Borel measurable.

The universal $\sigma$-algebra $\mathcal{U}$ is the intersection of the $\mu$-completions of the Borel $\sigma$-algebra on $X$, over the family of all probability measures $\mu$ on it. Its importance for us stems from the fact that $\mathcal{U}$-measurable functions can be integrated with respect to any probability measure, thus allowing us to treat them as if they were Borel measurable for most practical purposes.

A Suslin scheme on a set $X$ equipped with a family $\mathcal{G}$ of (not necessarily all of) its subsets, is a map $g: \cup_{n \in \mathbb{N}} \mathbb{N}^{n} \rightarrow \mathcal{G}$. The Suslin operation assigns to every Suslin scheme $g$ on $\mathcal{G}$ the set $A=\cup_{\sigma \in \mathbb{N}^{\mathbb{N}}} \cap_{n} g(\sigma(1), \ldots, \sigma(n))$. Two important facts about Suslin schemes in our context are the following: 1) $A$ is an analytic subset of a Polish space $X$ if and only if it is the result of some Suslin operation on the closed subsets of $X$, and 2) the universal $\sigma$-algebra is closed under the Suslin operation. It follows that analytic sets are universally measurable.

\section{REFERENCES}

[1] Charalambos D. Aliprantis and Kim C. Border, Infinite-dimensional analysis, SpringerVerlag, Berlin, second ed., 1999. A hitchhiker's guide.

[2] Erhan Bayraktar and Mihai Sîrbu, Stochastic Perron's method and verification without smoothness using viscosity comparison: obstacle problems and Dynkin games, ArXiv eprints, 1112.4904 (2012). to appear in Proceedings of the American Mathematical Society.

[3] - Stochastic Perron's method and verification without smoothness using viscosity comparison: the linear case, Proceedings of the American Mathematical Society, 140 (2012), pp. 3645-3654.

[4] - Stochastic Perron's method for Hamilton-Jacobi-Bellman equations, ArXiv e-prints, 1212.2170 (2012).

[5] Richard Bellman, Dynamic programming, Princeton University Press, Princeton, N. J., 1957.

[6] Dimitri P. Bertsekas and Steven E. Shreve, Stochastic optimal control, vol. 139 of Mathematics in Science and Engineering, Academic Press Inc. [Harcourt Brace Jovanovich Publishers], New York, 1978. The discrete time case. 
[7] - Existence of optimal stationary policies in deterministic optimal control, J. Math. Anal. Appl., 69 (1979), pp. 607-620.

[8] — Stochastic optimal control: The discrete time case, Athena Scientific, Belmont, Massachusetts, 1996. Reprint of the 1978 original.

[9] Jean-Michel Bismut, Théorie probabiliste du contrôle des diffusions, Mem. Amer. Math. Soc., 4 (1976), pp. xiii+130.

[10] David Blackwell, Discrete dynamic programming, Ann. Math. Statist., 33 (1962), pp. 719726.

[11] — Discounted dynamic programming, Ann. Math. Statist., 36 (1965), pp. 226-235.

[12] — Positive dynamic programming, in Proc. Fifth Berkeley Sympos. Math. Statist. and Probability (Berkeley, Calif., 1965/66), Vol. I: Statistics, Univ. California Press, Berkeley, Calif., 1967, pp. 415-418.

[13] David Blackwell, David Freedman, and Michael Orkin, The optimal reward operator in dynamic programming, Ann. Probability, 2 (1974), pp. 926-941.

[14] Vivek S. Borkar, Optimal control of diffusion processes, vol. 203 of Pitman Research Notes in Mathematics Series, Longman Scientific \& Technical, Harlow, 1989.

[15] Bruno Bouchard and Marcel Nutz, Weak dynamic programming for generalized state constraints, SIAM J. Control Optim., 50 (2012), pp. 3344-3373.

[16] Bruno Bouchard and Marcel Nutz, Arbitrage and Duality in Nondominated Discrete-Time Models, ArXiv e-prints, (2013). 1305.6008.

[17] Bruno Bouchard and Nizar TouzI, Weak dynamic programming principle for viscosity solutions, SIAM J. Control Optim., 49 (2011), pp. 948-962.

[18] Bruno Bouchard and Thanh Nam Vu, The obstacle version of the geometric dynamic programming principle: application to the pricing of American options under constraints, Appl. Math. Optim., 61 (2010), pp. 235-265.

[19] JaKŠa Cvitanić, WAlter Schachermayer, AND Hui WANG, Utility maximization in incomplete markets with random endowment, Finance and Stochastics, 5 (2001), pp. 237-259.

[20] Freddie Delbaen and Walter Schachermayer, The fundamental theorem of asset pricing for unbounded stochastic processes, Math. Ann., 312 (1998), pp. 215-250.

[21] Claude Dellacherie and Paul-André Meyer, Probabilities and potential. B, vol. 72 of North-Holland Mathematics Studies, North-Holland Publishing Co., Amsterdam, 1982. Theory of martingales, Translated from the French by J. P. Wilson.

[22] Nicole El Karoui, Les aspects probabilistes du contrôle stochastique, in Ninth Saint Flour Probability Summer School-1979 (Saint Flour, 1979), vol. 876 of Lecture Notes in Math., Springer, Berlin, 1981, pp. 73-238.

[23] Nicole El Karoui and Xiaolu Tan, Capacities, Measurable Selection and Dynamic Programming Part I: Abstract Framework, ArXiv e-prints, (2013). 1310.3363.

[24] - Capacities, Measurable Selection and Dynamic Programming Part II: Application in Stochastic Control Problems, ArXiv e-prints, (2013). 1310.3364.

[25] Stewart N. Ethier and Thomas G. Kurtz, Markov processes, Wiley Series in Probability and Mathematical Statistics: Probability and Mathematical Statistics, John Wiley \& Sons Inc., New York, 1986. Characterization and convergence.

[26] Wendell H. Fleming And H. Mete Soner, Controlled Markov processes and viscosity solutions, vol. 25 of Applications of Mathematics (New York), Springer-Verlag, New York, 1993.

[27] — Controlled Markov processes and viscosity solutions, vol. 25 of Stochastic Modelling and Applied Probability, Springer, New York, second ed., 2006.

[28] Hans Föllmer and Philip Protter, Local martingales and filtration shrinkage, ESAIM Probab. Stat., 15 (2011), pp. S25-S38.

[29] Olav Kallenberg, Foundations of modern probability, Probability and its Applications (New York), Springer-Verlag, New York, second ed., 2002.

[30] Alexander S. Kechris, Classical descriptive set theory, vol. 156 of Graduate Texts in Mathematics, Springer-Verlag, New York, 1995.

[31] Ashok P. Maitra and William D. Sudderth, Discrete gambling and stochastic games, vol. 32 of Applications of Mathematics (New York), Springer-Verlag, New York, 1996.

[32] Marcel Nutz, Random G-expectations. to appear in Annals of Applied Probability, 2012.

[33] Marcel Nutz and Ramon van Handel, Constructing sublinear expectations on path space, 
Stochastic Process. Appl., 123 (2013), pp. 3100-3121.

[34] Philip E. Protter, Stochastic integration and differential equations, vol. 21 of Applications of Mathematics (New York), Springer-Verlag, Berlin, second ed., 2004. Stochastic Modelling and Applied Probability.

[35] Daniel Revuz and Marc Yor, Continuous martingales and Brownian motion, vol. 293 of Grundlehren der Mathematischen Wissenschaften [Fundamental Principles of Mathematical Sciences], Springer-Verlag, Berlin, third ed., 1999.

[36] Steven E. Shreve and Dimitri P. Bertsekas, Universally measurable policies in dynamic programming, Math. Oper. Res., 4 (1979), pp. 15-30.

[37] H. Mete Soner and Nizar Touzi, Dynamic programming for stochastic target problems and geometric flows, J. Eur. Math. Soc. (JEMS), 4 (2002), pp. 201-236.

[38] — Stochastic target problems, dynamic programming, and viscosity solutions, SIAM J. Control Optim., 41 (2002), pp. 404-424.

[39] Shashi M. SRivastava, A course on Borel sets, vol. 180, Springer, New York, 1998.

[40] Ralph E. Strauch, Negative dynamic programming, Ann. Math. Statist., 37 (1966), pp. 871890.

[41] Christophe Stricker, Quasimartingales, martingales locales, semimartingales et filtration naturelle, Probability Theory and Related Fields, 39 (1977), pp. 55-63.

[42] Daniel W. Stroock and S. R. Srinivasa Varadhan, Multidimensional diffusion processes, Classics in Mathematics, Springer-Verlag, Berlin, 2006. Reprint of the 1997 edition.

[43] Veeravali S. Varadarajan, Groups of automorphisms of Borel spaces, Trans. Amer. Math. Soc., 109 (1963), pp. 191-220.

[44] Abraham Wald, Statistical Decision Functions, John Wiley \& Sons Inc., New York, N. Y., 1950.

[45] Jiongmin Yong And Xun Yu Zhou, Stochastic controls, vol. 43 of Applications of Mathematics, Springer-Verlag, New York, 1999. 